\title{
Evaluation of information transfer between beef producers and Extension Agents in West Virginia
}

Travis J. Cullen

West Virginia University

Follow this and additional works at: https://researchrepository.wvu.edu/etd

\section{Recommended Citation}

Cullen, Travis J., "Evaluation of information transfer between beef producers and Extension Agents in West Virginia" (2010). Graduate Theses, Dissertations, and Problem Reports. 2990.

https://researchrepository.wvu.edu/etd/2990

This Thesis is protected by copyright and/or related rights. It has been brought to you by the The Research Repository @ WVU with permission from the rights-holder(s). You are free to use this Thesis in any way that is permitted by the copyright and related rights legislation that applies to your use. For other uses you must obtain permission from the rights-holder(s) directly, unless additional rights are indicated by a Creative Commons license in the record and/ or on the work itself. This Thesis has been accepted for inclusion in WVU Graduate Theses, Dissertations, and Problem Reports collection by an authorized administrator of The Research Repository @ WVU. For more information, please contact researchrepository@mail.wvu.edu. 


\title{
Evaluation of Information Transfer between Beef Producers and Extension Agents in West Virginia
}

\author{
Travis J. Cullen
}

\author{
Thesis submitted to the \\ Davis College of Agriculture, Natural Resources and Design \\ at West Virginia University \\ in partial fulfillment of the requirements \\ for the degree of \\ Master of Science \\ in \\ Agricultural and Extension Education \\ Deborah A. Boone, Ph.D., Chair \\ Harry N. Boone, Jr., Ph.D. \\ Jean M. Woloshuk, Ed.D.
}

Division of Resource Management

\author{
Morgantown, West Virginia \\ 2010
}

Keywords: Extension, Beef Producers, West Virginia, Extension Agents, Information Transfer 


\section{ABSTRACT \\ Evaluation of Information Transfer between Beef Producers And Extension Agents in West Virginia}

\section{Travis J. Cullen}

The purpose of this study was to determine how information passes between beef producers and Extension Agents in West Virginia in the areas of teaching methods and program advertisement. A descriptive research design was used to collect the data for this study. The target population was all beef producers and the Agriculture and Natural Resource Extension Agents in West Virginia. The study found that beef producers and Extension Agents tend to be in agreement on both the methods of advertising and teaching. However, the programs beef producers desire differs from those the West Virginia University Extension Service offers. 


\title{
DEDICATION
}

This thesis is dedicated to the important people in my life who have always been supportive of everything I have done:

\author{
Papaw and Mamaw
}

Dad and Cindy 


\section{ACKNOWLEDGEMENTS}

Writing a thesis takes a lot of time, work, and help from many people, I would like to thank those who have helped me achieve this goal.

To Dr. Debby Boone, you have the patience of a saint. While working on my Master's degree you have answered thousands of questions about everything. Thank you for always taking the time out of your busy day to answer those questions, and having the confidence in me to reach this goal.

To Dr. Harry Boone, without you I could not have begun the process of writing a thesis. Thank you for giving us the foundation and knowledge of writing a thesis, and of course assisting in analyzing my data. Without you I would probably still be trying to figure out how to write a thesis.

To Dr. Jean Woloshuk, you started my goals of becoming an Extension Agent. Thank you for giving me the opportunity to begin my Extension career with you. The knowledge I have learned from you will be with me for a lifetime. Thank you for serving on my committee and providing your knowledge in creating my instrument.

To Alice Compton, you are the heart of the Ag Ed Department, where would I be without you? This project would have never been completed if it weren't for your contributions and efforts. Thank you for all your help.

To my family, can you believe it's over? Even when I doubted myself you always had the confidence in me. Thank you for always believing in me and knowing this venture was going to benefit me beyond words even when I didn't. Your words of support and understanding and your guidance will always be with me.

The many people throughout my life that have influenced who I am today, I could never begin to list or name. I have been blessed by each and every one of you and I am who I am today because of you all. 


\section{TABLE OF CONTENTS}

DEDICATION

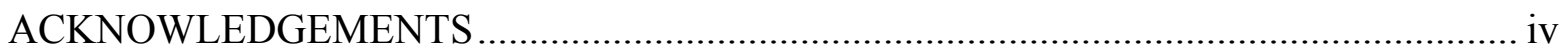

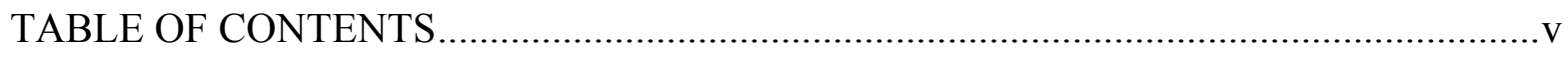

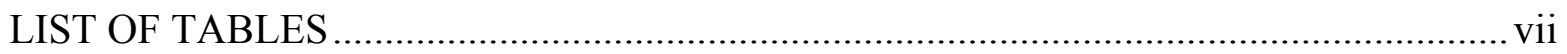

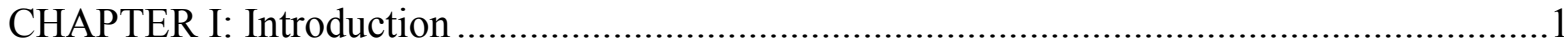

Purpose and Objectives ......................................................................................... 2

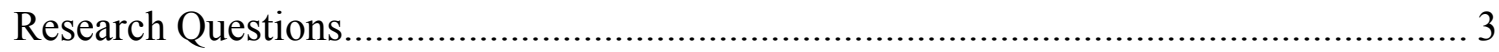

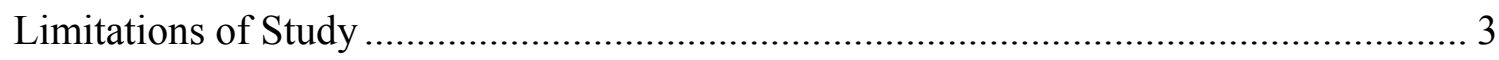

CHAPTER II: Review Of Literature ........................................................................4

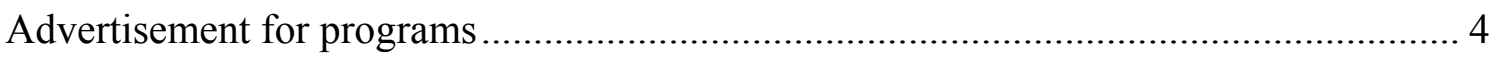

Teaching Methods Used During Programs ........................................................... 5

West Virginia Beef Producers and Technology ....................................................... 5

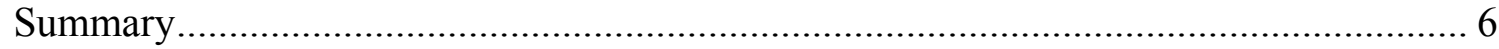

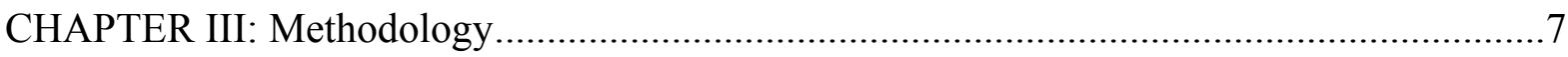

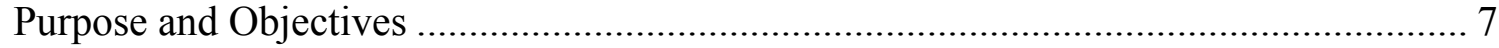

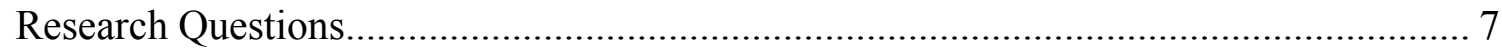

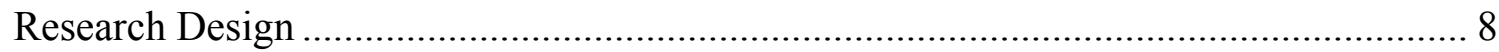

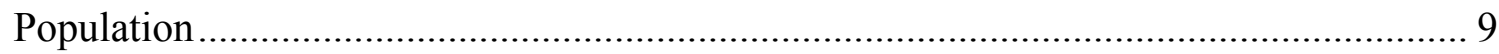

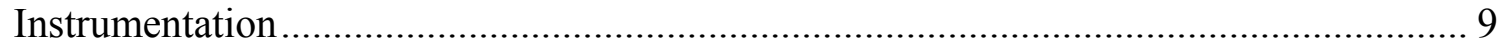

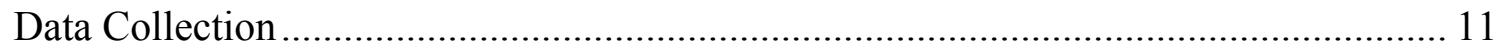

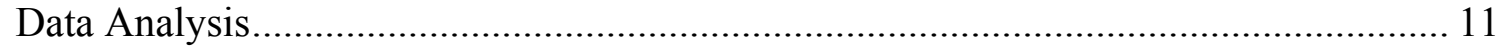

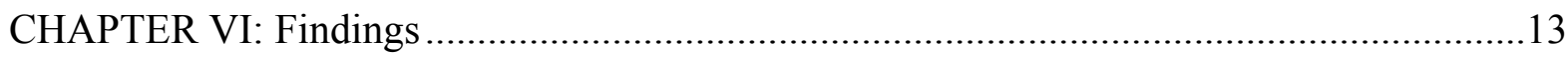

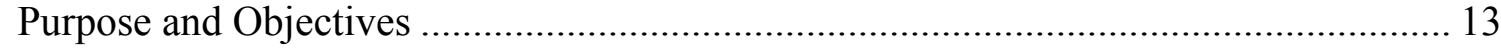

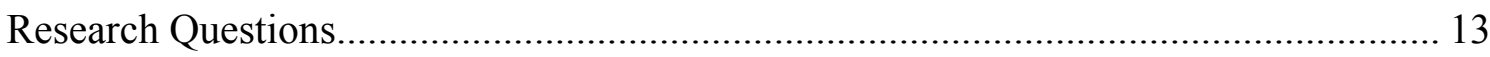

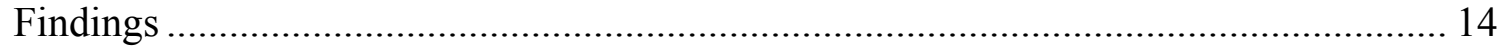

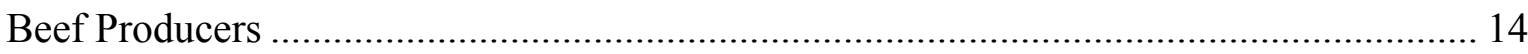

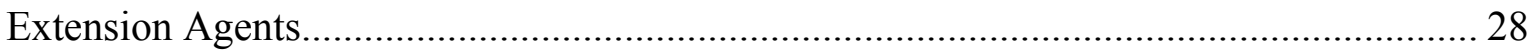

Comparisons of Extension Agents and West Virginia Beef producers ........................... 40 
CHAPTER V: Summary, Conclusions, Recommendations ............................................53

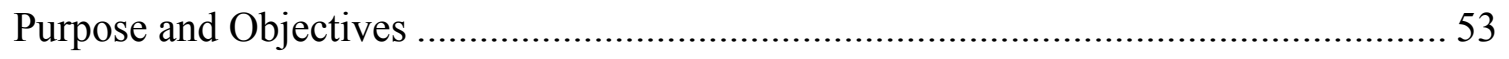

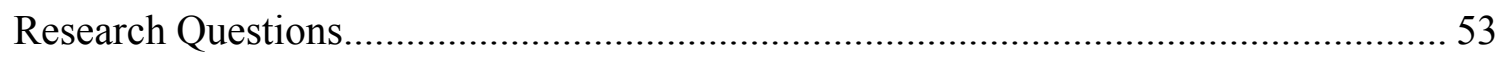

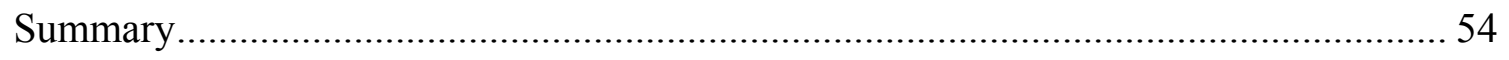

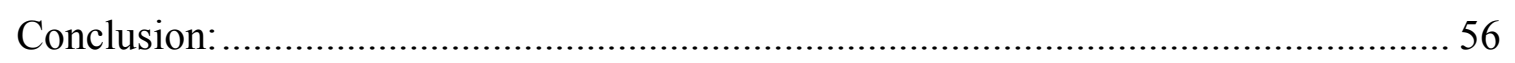

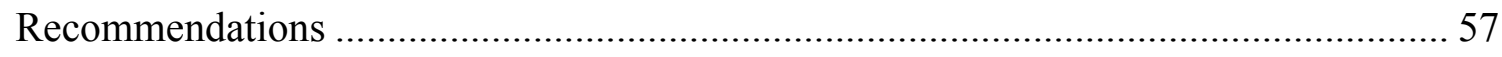

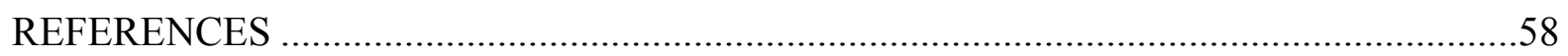

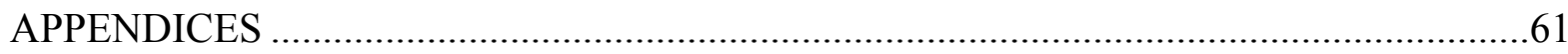

APENDIX A: First Mailing Cover Letter: Extension Agent.............................................62

APENDIX B: Second Mailing Cover Letter: Extension Agent .......................................64

APPENDIX C: First Mailing Cover Letter: Beef Producer...............................................66

APPENDIX D: Second Mailing Cover Letter: Beef Producer ...........................................68

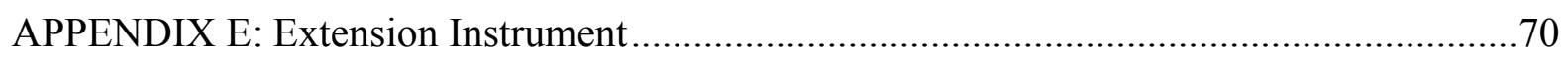

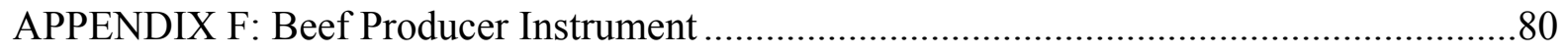

APPENDIX G: Extension Agent Open Ended Responses ............................................90

APPENDIX H: Beef Producer Open Ended Responses ................................................92

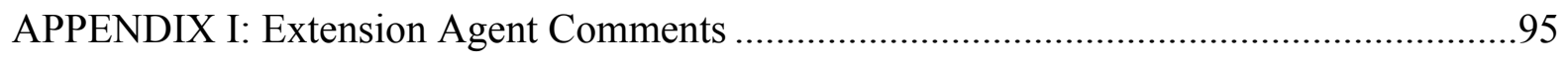

APPENDIX J: Beef Producer Comments ....................................................................... 97

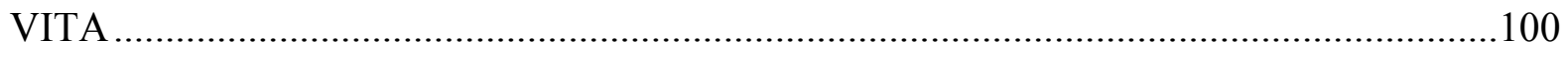




\section{LIST OF TABLES}

Number Title Page

1 Evaluation of Local Extension Agent and WVU Extension Programs …........17

2 West Virginia Beef Producers Preferences to Receive Advertisements about Extension Programs ..............................................................................19

3 Teaching Methods Beef Producers Prefer to Learn …………………….........20

$4 \quad$ West Virginia Beef Producers Interest in West Virginia University

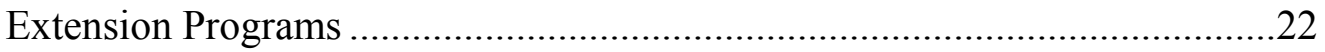

$5 \quad$ Number of Beef Extension Programs Attended Per Year as Reported

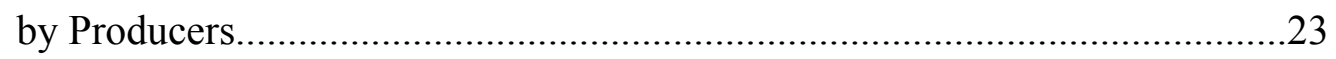

$6 \quad$ Percentage of Extension Programming that are Beef Programs ......................23

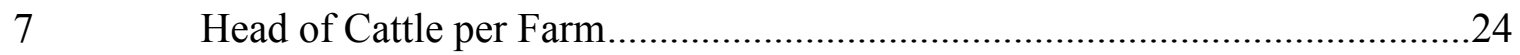

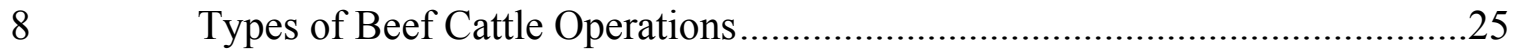

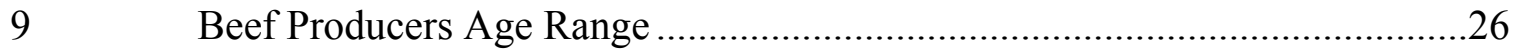

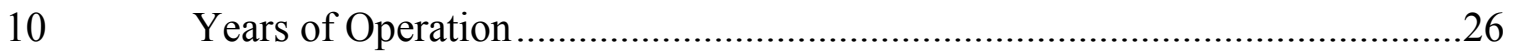

11 Local Extension Agent, who Handles Beef Programming …………..............27

12 How do Beef Producers get Answers to Their Beef Questions .......................28

13 Self Evaluation of WVU Extension Agents and Their Beef Programs ............31

14 Methods of Advertisement WVU Extension Agents Use.................................32

15 Teaching Methods Extension Agents Prefer to Uses to Deliver Beef

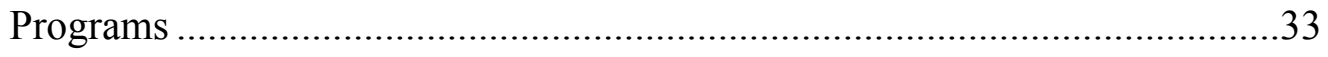

16 Beef Programs Offered by West Virginia University Extension Agents .........34

$17 \quad$ Number of Beef Extension Programs, Agents Offer per Year .........................35

18 Average attendance of Extension Programs per Year …………………….......36

19 Average Number of West Virginia Beef Producers Worked with Each

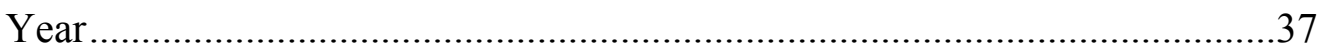

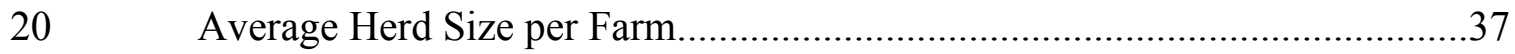


22 Average Age of Farmers in Their County ...... .38

23 Average Years of Beef Cattle Operation in Their County.............................39

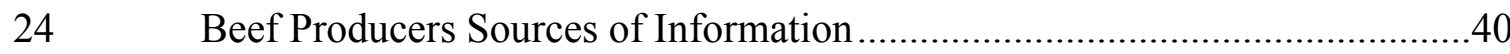

25 Comparison of the Mean Scores of the WVU Extension Agent as a

Source of Information

26 Comparison of the Mean Scores of the Veterinarians as a Source of Information

27 Comparison of the Mean Scores of the Neighbor as a Source of

Information

28 Comparison of the Mean Scores of the Extension Specialist as a

Source of Information

29 Comparison of Mean Scores of a Farm Store Employee as a Source of

Information .....

30 Comparison of Mean Scores of Other Sources of Information

31 Comparison of Advertisement Methods ...................................................48

32 Comparison of Teaching Methods .........................................................49

33 Comparison of Extension Programming .......................................................51 


\section{CHAPTER I}

\section{Introduction}

The West Virginia beef industry ranks $38^{\text {th }}$ in the nation (National Agricultural Statistics Service, 2007) with nearly 12,000 farms operating in all 55 counties (Census of Agriculture, 2007). In beef production, like any other agricultural operation, questions arise. In today's society those questions may be answered using several resources which include a neighbor, a veterinarian, or a farm store employee. However there is one other resource that has been available for over a hundred years, the local West Virginia University Extension Agent.

In 1914 the US Congress passed the Smith-Lever Act which established the Cooperative Extension Service (CES) (Seevers, Graham \& Conklin, 2007). The CES is in a cooperative of three partners: the National Institute of Food and Agriculture (NIFA), state government and state land grant universities, and county governments (National Institute of Food and Agriculture, 2009). CES's mission is “...to aid in diffusing among the people of the United States useful and practical information on subjects relating to agriculture, uses of solar energy with respect to agriculture, home economics, and rural energy, and to encourage the application of the same" (Smith-Lever Act, 2002, sec. 1).

West Virginia University Extension Agents are available to assist people with topics ranging from families and health to $4-\mathrm{H}$ and agriculture. With technology increasing, Extension Agents continue to adopt new methods of marketing their programs. In a study by Neehouse (2005) West Virginia University Extension Agents ranked using the Internet to transfer information third after newsletters and newspapers. Richardson \& Mustian (1988) cautioned that Extension should avoid moving too rapidly 
into newer impersonal forms of communications to meet informational needs of its agricultural audiences. Though agents are using newer forms of technology to get their information out to the community, it is important to not overlook those clients that may not have the newest forms of technology to receive that information. A study at Michigan State University found that only $10 \%$ of the farmers in their study received information from the Internet (Suvedi, Lapinski \& Campo, 2000).

West Virginia's Beef Industry is very diverse from cow/calf operations to seedstock farms. In the 2007 Census of Agriculture there were approximately 12,000 farms with approximately 400,000 beef cattle while the average age of the West Virginia farmer was 58.1 years of age. With West Virginia's beef industry so diverse, how are Extension Agents reaching out and adapting their programs to the individual beef operations? Do Extension Agents offer programs for both small and large scale operations? By understanding what the beef producers' preferences are in the way of programs, teaching methods, and information transfer, Extension Agents will better adapt their programs, communication methods, and in return increase the average attendance for programs relating to West Virginia's beef industry.

\section{Purpose and Objectives}

The purpose of this study was to determine whether information from the West Virginia University Extension Service being disseminated to the West Virginia beef producers through appropriate programming and advertising. The primary objective of this study was to determine whether Extension Agents are offering programs beef producers want in their counties. The research focused on program advertising, teaching methods, and program offerings. 


\section{Research Questions}

The objectives of study were reflected in the following research questions:

1. What methods are West Virginia University Extension Agents using to inform beef producers about upcoming programs and new technologies relating to the beef industry?

2. How do beef producers prefer to hear about Extension programs?

3. What methods do West Virginia University Extension Agents use to teach their programs?

4. What are the methods by which West Virginia beef producers prefer to learn?

5. What beef production programs are West Virginia University Extension Agents offering in the state?

6. What programs would the beef producers like to see West Virginia University Extension Service offer in their counties?

7. Are West Virginia University Extension Agents effectively communicating with their county beef producers?

\section{Limitations of Study}

The study was limited to beef producers that have participated in the following: Weston \& South Branch Livestock Market Sales, Southern Bull Test, Beef Quality Assurance Program, members of the West Virginia Cattleman's Association, and State Livestock Roundup. 


\section{CHAPTER II}

\section{Review of Literature}

The Cooperative Extension Service (CES) is made up of the United States Department of Agriculture (USDA), land-grant universities, and county administrative units (Seevers, Graham \& Conklin, 2007). From the beginning the goal of the CES has been to use technology and information to assist people and improve their quality of life (Houghton, Arrington, \& Bradshaw, 1994).

\section{Advertisement for Programs}

Warner (1996) found that when asked if people had ever heard of the CES, only $45 \%$ said they had; this was an increase of $40 \%$ from 1982 . When people were asked if they were aware of Extension agriculture programs or 4-H programs, 4-H had the greatest response of $69 \%$, where only half the respondents were aware of agriculture programs. Farmers have also viewed Extension agriculture research and programs skeptically (Gerber, 1992). A study by Habeeb, Birkenholz, and Weston (1987) reported that persons who are more innovative tend to be less satisfied with Extension's information, specialists, and agriculture education programs. Before a program can become successful, people must be made aware of the program itself. Extension programs can be marketed using a four step plan. Step one, conduct audience inventories, step two define your goals and specify your objectives, step three is to decide on the nature of your message, and finally step four is to decide on the appropriate media (Nehiley, 2001). Burrows (2008) found it was highly effective to promote Extension programs by setting up a booth at the local farmers market. The booth provided a high profile, but didn't require a lot of expense. Rexroad (2002) found that most people become aware of other 
programs through attendance at other Extension programs, followed by referrals from friends and through reading newspapers.

\section{Teaching Methods Used During Programs}

An Extension Agent's job is to provide information for their clients about the newest and latest agricultural practices. A study by Peters (1998) found four key factors that lead to adoption, including credibility, mutual trust, farm demonstrations, and communications. Though the agents must use these factors, the actual rate of adoption varies; although there are several methods which can be used in teaching adults. It is essential to use the right teaching methods, Riesenberg \& Gor (1989) found that teaching methods should vary depending upon the age of the farmer, size of operation, and the educational status of the farmer. Younger farmers, as well as those with a college education, prefer to use computers to learn. Richardson and Mustian (1988) found that newsletters, followed by farm visits and meetings were the most popular teaching methods among farmers in North Carolina. Chizari, Karbasioun, \& Linder (1998) found that demonstrations were the most effective way to teach adults, where the least favorable methods were lecture and presentations. Nelson (2008) also found that demonstrations were the most preferred method of teaching for dairy producers in Pennsylvania. Factors that often affect the learning process of the clientele are the lack of facilities or teaching equipment (Chizari et al, 1998). Gamon, Harrold and Creswell (1994) looked at the rates

of acceptance of new practices and found there were no differences between farmers who do and do not attend programs.

\section{West Virginia Beef Producers and Technology}

Over the last decade the way farmers can obtain information has changed rapidly. A study conducted by Iddings and Apps (1990) focused on how farmers felt about using 
computers. Their study found that farmers felt they were too old to learn the skills to use a computer. On the contrary, Findlay, Zabawa, Morris, \& Oben (1993) found that farmers were willing to learn more about computers, if training was available. A study conducted by Batte (2004) focused on the rate farmers have adopted the use of computers. Batte also found that the rate of adoption varied among the following demographics: age, gross sales, and education. Farmers under the age of 50 (54\%) were more likely to use a computer, where farmers over $50(22 \%)$ were less likely to have computers on their farms. It was also found that 72 percent of farms with a gross sale of $\$ 500,000$ or more used computers in their operations, where 24 percent of farms with sales under $\$ 250,000$ (21.4\%) used computers on their farms. Education also had an influence on computer adoption. Sixty-seven percent of farmers with post high school education were computer users (Batte, 2004).

The West Virginia beef industry is worth an estimated 1.6 million dollars, made up of more than 400,000 cattle on 12,000 farms operating in all fifty-five counties (National Agricultural Statistic Service, 2008). West Virginia's average size farm is about 157 acres, down nine percent from 172 acres in 2002. While the average farmer's age in West Virginia is 59 years of age, using the computer as a primary source may be out of the question (Census of Agriculture, 2007).

\section{Summary}

Studies relating to the overall themes of the research questions proposed for this study vary in age. Research on beef producer's preferences on delivery methods has been conducted over several years, while research relating to how Extension Agents prefer to transfer their information has been conducted is more recent. No studies were found that link beef producer's preferences to Extension Agent's preferences. 


\section{CHAPTER III}

\section{Methodology}

\section{Purpose and Objectives}

The purpose of this study was to determine whether information from the West Virginia University Extension Service being disseminated to the West Virginia beef producers through appropriate programming and advertising. The primary objective of this study was to determine whether the Extension Agents are offering the programs beef producers want in their counties. The research focused on program advertising, teaching methods, and program offerings.

\section{Research Questions}

The objectives of study were reflected in the following research questions:

1. What methods are West Virginia University Extension Agents using to inform beef producers about upcoming programs and new technologies relating to the beef industry?

2. How do beef producers prefer to hear about Extension programs?

3. What methods do West Virginia University Extension Agents use to teach their programs?

4. What are the methods by which West Virginia beef producers prefer to learn?

5. What beef production programs are West Virginia University Extension Agents offering in the state?

6. What programs would the beef producers like to see West Virginia University Extension Service offer in their counties?

7. Are West Virginia University Extension Agents effectively communicating with their county beef producers? 


\section{Research Design}

A descriptive research design was chosen to evaluate the research questions for the study. According to Ary, Jacobs, Razavieh, Sorensen (2006), surveys allow the researcher to measure the attitudes and opinions of the respondents to collect information from a sample of the target population. Surveys allow the population to be reached regardless of location.

With survey research there are five errors that need to be controlled. To avoid frame error for the beef producers a list was created from six participant lists: the Southern Bull Test, Beef Quality Assurance Program, members of the West Virginia Cattleman's Association mailing list, participants in the South Branch and Weston livestock markets, and State Livestock Roundup participants. The beef producer list was then cleared of all duplicates. To control frame error for the Extension Agents, the official list was obtained from the West Virginia University West Virginia University Extension Service. To avoid sample error producers were randomly selected using SPSS statistical software; and a census was taken of all agents responsible for Agriculture and Natural Resources, or in charge for single agent counties.

Selection error was avoided by randomly selecting additional beef participants. These participants were added to the participant list if a duplicate was found. To control measurement error the instruments were tested for reliability and validity.

To avoid non-response error, a comparison was conducted to find the differences between early and late respondents using the Pearson Chi-Square. The variables included herd size, years of operation, and age. The Chi-Square showed no significant differences between the variables, therefore generalizations for both beef producers and Extension Agents could be made for the total population. 


\section{Population}

The target population for this study was Extension Agents responsible for Agriculture and Natural Resources programming and those Extension Agents in single agent charge single agent counties. A census was conducted of 53 agents responsible for Agriculture \& Natural Resources programming for the 55 counties.

The second target population for this study was all beef producers in West Virginia. Due to the lack of availability of an official list of beef producers for state of West Virginia, the accessible population was selected from a compiled list of participants in the Southern Bull Test, Beef Quality Assurance Program, members on the West Virginia Cattleman's Association mailing list, participants in the South Branch and Weston livestock markets, and State Livestock Roundup ( $\mathrm{N}=4600)$. The Krejcie and Morgan guidelines (1960) were used to determine the sample size of the beef producers $(n=365)$.

\section{Instrumentation}

Two different instruments were used in this study; one was designed for beef producers and one for WVU Extension Agents in West Virginia. The surveys were adapted from two instruments created by Nelson (2008) and used with Extension Agents and dairy producers in Pennsylvania. Because the attitudes were to be determined and the size of the population, the most appropriate type of survey was the survey of intangibles. This type of survey allows for public opinions without coming into contact with the participants.

The Extension Agent instrument was broken into three parts. The first part consisted of Likert-type questions designed to determine the agents' performance, advertising and teaching methods, and the type of beef programs they offer. The 
questions had six responses available. The responses included strongly agree, moderately agree, agree, disagree, moderately disagree, and strongly disagree. The neutral response was purposely omitted as recommended by Ary et al., (2006) to ensure that an opinion was given for each question to determine attitudes. The second section consisted of a set of open-ended questions used to determine Extension Agent's perception of the demographics of the beef producers in their area and beef producers' use of the West Virginia University Extension Service. The final section of the instrument was for comments.

The beef producers' instrument was also broken into three parts. Like the Extension agent instrument the first part consisted of Likert-type questions. These questions were designed to evaluate their county's Extension Agent, how they receive information from their agent and producers preferences for how they receive information, as well as what programs, topics, and teaching methods they prefer be used. These questions had six or seven responses including strongly agree, moderately agree, agree, disagree, moderately disagree, strongly disagree, and not applicable. Again the neutral response was omitted to ensure an opinion. The second section consisted of open-ended type questions used to determine the producers use the West Virginia University Extension Service and their farm's demographics. The final section of the instrument was for comments.

\section{Validity of the Instrument}

The instruments were presented to a panel of faculty members in the Agricultural and Extension Education department and Extension Specialists at West Virginia University to establish its content and face validity. Each individual on the panel had 
extensive teaching or Extension experience. The panel determined that the instruments had content and face validity.

\section{Reliability of the Instrument}

The reliability of the Extension Agents' instrument was determined using the final data set. Because the data consisted of nominal and ordinal scale responses, the Spearman Brown split half statistic was used to establish the instrument's reliability. The reliability was found to be exemplary with the coefficient of 0.95 (Robinson, Shaver, \& Wrightsman, 1991). The instrument was established as a reliable measure.

The reliability of the beef producers' instrument was determined using the final data set. Because the data consisted of nominal and ordinal scale responses, the Spearman Brown split half statistic was used to establish the instrument's reliability. Reliability was found to be exemplary with a coefficient of 0.86 (Robinson, Shaver, \& Wrightsman, 1991). The instrument was established as a reliable measure.

\section{Data Collection}

Dillman's Tailored Design Method (2007) was adopted for this study. A packet was mailed on January 11, 2010 with the following: a cover letter explaining the purpose of study, the instrument, and a pre-paid self-addressed envelope. Participants were given a deadline of January 25, 2010 to return the questionnaire. A second mailing was sent to all non-respondents on January 29, 2010. This packet consisted of a second cover letter, a second instrument, and a pre-paid self-addressed envelope. These individuals were given a second deadline of February 12, 2010.

\section{Data Analysis}

The data were entered into an Excel spreadsheet. The data were then transferred to SPSS for analysis, and the significance level was set $a$ priori at $\leq .05$ for all statistical 
tests. Frequency tables were generated for both instruments. A difference of means $t$-test was then used to compare means for beef producers and Extension Agents. 


\section{CHAPTER VI}

\section{Findings}

\section{Purpose and Objectives}

The purpose of this study was to determine whether information from the West Virginia University Extension Service is being disseminated to the West Virginia beef producers through appropriate programming and advertising. The primary objective of this study was to determine whether the Extension Agents are offering the programs beef

producers want in their counties. The research focused on program advertising, teaching methods, and program offerings.

\section{Research Questions}

The objectives of study were reflected in the following research questions:

1. What methods are West Virginia University Extension Agents using to inform beef producers about upcoming programs and new technologies relating to the beef industry?

2. How do beef producers prefer to hear about Extension programs?

3. What methods do West Virginia University Extension Agents use to teach their programs?

4. What are the methods by which West Virginia beef producers prefer to learn?

5. What beef production programs are West Virginia University Extension Agents offering in the state?

6. What programs would the beef producers like to see West Virginia University Extension Service offer in their counties?

7. Are West Virginia University Extension Agents effectively communicating with their county beef producers? 


\section{Findings}

The first target population for this study consisted of beef producers in West Virginia. The sample population of 365 was randomly selected. Ten were returned as undeliverable, five were returned as deceased, and eight producers had sold out, or went out of business making the sample population 342. Out of the 342 questionnaires 142 $(42 \%)$ were returned.

The second target population for this study consisted of an Extension Agent from each county in West Virginia County. The sample population was a census of all agents responsible for Agriculture and Natural Resources, or agents in charge of single agent counties $(\mathrm{N}=51)$. Five counties in West Virginia reported they do not offer beef programs making the total sample size of Extension Agents offering beef programs 46. Of the 46 questionnaires, $40(87 \%)$ were returned.

\section{Beef Producers}

Beef producers were asked to evaluate their West Virginia University (WVU) Extension Agent's beef cattle knowledge and programs related to beef production. One hundred fourteen participants $(90.4 \%)$ were in agreement that their WVU Extension Agent offers programs in beef production. Fifty-seven (45.2\%) strongly agreed, 45 (35.7\%) agreed, and 12 (9.5\%) moderately agreed their WVU Extension Agent offered beef related programs. Six respondents $(4.8 \%)$ disagreed, three $(2.4 \%)$ moderately disagreed, and two (1.6\%) strongly disagreed to having beef programs offered by their WVU Extension Agent. One participant (.8\%) selected not applicable (see Table 1).

One hundred four participants $(84.8 \%)$ agreed in some way that their WVU Extension Agents were able to answer their beef related questions. Forty-five participants (36\%) strongly agreed, 44 (35.2\%) agreed, and $17(13.6 \%)$ moderately 
agreed. Seventeen participants (13.6\%) responded that their WVU Extension Agents could not answer their beef related questions, with 10 participants $(8.0 \%)$ who disagreed, four (3.2\%) moderately disagreed, and three (3.4\%) strongly disagreed. Two participants (1.6\%) responded not applicable (see Table 1).

West Virginia beef producers were asked if their WVU Extension Agents returned their phone calls in a timely manner. Forty-eight respondents (38.1\%) strongly agreed, $31(24.6 \%)$ agreed, and $23(28.3 \%)$ moderately agreed with the statement. Three respondents $(2.4 \%)$ were in some sort of disagreement. Two respondents $(1.6 \%)$ disagreed and one (.8\%) moderately disagreed that their WVU Extension Agents did not return their calls in a timely manner. Twenty-one (16.7\%) participants selected not applicable (see Table 1).

When asked if their WVU Extension Agents returned email in timely manner, 68 (66.7\%) responded not applicable with 42 of respondents stating they did not have email. Thirty-two (31.4\%) respondents were in some form of agreement with $15(14.7 \%)$ strongly agreeing, $12(11.8 \%)$ respondents agreeing, and five respondents moderately agreeing. Two (2.0\%) respondents disagreed that their WVU Extension Agents returned their email in a timely manner (see Table 1).

When asked if West Virginia beef producers attended Extension beef programs in their county, 96 (76.8\%) participants were in some form of agreement. Forty-two (33.6\%) of the participants agreed, 39 (31.2\%) of the participants strongly agreed, and 15 $(12.0 \%)$ participants moderately agreed. Sixteen $(12.8 \%)$ were in some form of disagreement about attending Extension beef programs in their county. Eleven (8.8\%) 
participants disagreed, four (3.2\%) moderately disagreed, and one (0.8\%) participant strongly agreed. Thirteen (10.4\%) participants selected not applicable (see Table 1).

West Virginia Beef producers were asked if Extension programs provided adequate information which would influence them to change their practices. Forty-eight (37.5\%) respondents agreed, $39(31.2 \%)$ respondents strongly agreed, and $15(12.0 \%)$ moderately agreed. Eleven $(8.6 \%)$ respondents were in some form of disagreement that Extension programs provided adequate information which would influence them to change their practices. Six (4.7\%) respondents disagreed, three (2.3\%) respondents strongly disagreed, and two (1.6\%) respondents moderately disagreed. Seven (5.5\%) respondents selected not applicable (see Table 1).

When asked if their local Extension agent is knowledgeable about beef production, $43(33.9 \%)$ producers strongly agreed, 40 (31.5\%) producers agreed, and 19 (15.0\%) producers moderately agreed. Twenty $(15.7 \%)$ producers were in some form of disagreement and felt their Extension agent was not knowledgeable about beef production. Twelve (9.4\%) producers disagreed, five (3.9\%) producers strongly disagreed, and three (2.4\%) producers moderately disagreed. Five (3.9\%) producers selected not applicable (see Table 1). 
Table 1

Evaluation of Local Extension Agent and WVU Extension Programs

\begin{tabular}{|c|c|c|c|c|c|c|c|c|c|c|c|c|c|c|}
\hline & \multicolumn{2}{|c|}{ Not applicable } & \multicolumn{2}{|c|}{$\begin{array}{l}\text { Strongly } \\
\text { Disagree }\end{array}$} & \multicolumn{2}{|c|}{$\begin{array}{l}\text { Moderately } \\
\text { Disagree }\end{array}$} & \multicolumn{2}{|c|}{ Disagree } & \multicolumn{2}{|c|}{ Agree } & \multicolumn{2}{|c|}{$\begin{array}{c}\text { Moderately } \\
\text { Agree }\end{array}$} & \multicolumn{2}{|c|}{ Strongly Agree } \\
\hline & $\mathrm{N}$ & $\%$ & $\mathrm{~N}$ & $\%$ & $\mathrm{~N}$ & $\%$ & $\mathrm{~N}$ & $\%$ & $\mathrm{~N}$ & $\%$ & $\mathrm{~N}$ & $\%$ & $\mathrm{~N}$ & $\%$ \\
\hline $\begin{array}{l}\text { Offers programs in beef } \\
\text { production }\end{array}$ & 1 & .8 & 2 & 1.6 & 3 & 2.4 & 6 & 4.8 & 45 & 35.7 & 12 & 9.5 & 57 & 45.2 \\
\hline $\begin{array}{l}\text { Able to answer beef } \\
\text { production questions }\end{array}$ & 2 & 1.6 & 3 & 2.4 & 4 & 3.2 & 10 & 8.0 & 44 & 35.2 & 17 & 13.6 & 45 & 36.0 \\
\hline $\begin{array}{l}\text { Returns telephone calls } \\
\text { in a timely manner }\end{array}$ & 21 & 16.7 & 0 & .0 & 1 & .8 & 2 & 1.6 & 31 & 24.6 & 23 & 18.3 & 48 & 38.1 \\
\hline $\begin{array}{l}\text { Returns emails in a } \\
\text { timely manner }\end{array}$ & 68 & 66.7 & 0 & .0 & 0 & .0 & 2 & 2.0 & 12 & 11.8 & 5 & 4.9 & 15 & 14.7 \\
\hline $\begin{array}{l}\text { I attend Extension beef } \\
\text { programs in my county }\end{array}$ & 13 & 10.4 & 1 & .8 & 4 & 3.2 & 11 & 8.8 & 42 & 33.6 & 15 & 12.0 & 39 & 31.2 \\
\hline $\begin{array}{l}\text { Extension programs } \\
\text { provide adequate } \\
\text { information }\end{array}$ & 7 & 5.5 & 3 & 2.3 & 2 & 1.6 & 6 & 4.7 & 48 & 37.5 & 30 & 23.4 & 32 & 25.0 \\
\hline $\begin{array}{l}\text { Local Extension agent is } \\
\text { knowledgeable about } \\
\text { beef production }\end{array}$ & 5 & 3.9 & 5 & 3.9 & 3 & 2.4 & 12 & 9.4 & 40 & 31.5 & 19 & 15.0 & 43 & 33.9 \\
\hline
\end{tabular}


West Virginia beef producers were asked how they preferred to receive information about upcoming Extension programs. The top five methods of advertisement beef producers preferred were: mail with 124 (98.4\%) respondents; newsletters with 118 (96.7\%) respondents; flyers with $96(87.3 \%)$ respondents; personal visits with 96 (86.5\%) respondents; and newspapers with 96 (84.2\%) respondents. Respondents also showed interest in phone (70.6\%), email (66.7\%), Internet (66.7\%), television (68.9\%), and radio (64.8\%). Three respondents indicated other selections writing in farm visits, this would be the same as personal visits (see Table 2).

West Virginia beef producers were also asked how they received information about upcoming Extension programs. The top five methods of advertisement by which beef producers receive information were: mail with 109 (97.3\%) respondents; newsletters with $89(84.8 \%)$ respondents; word of mouth with 84 (82.4\%) respondents; newspapers with 79 (77.5\%) respondents; flyers with 74 (77.9\%) respondents; and personal visits with 54 (58.7\%) respondents. Respondents also indicated they received information about upcoming programs by phone (56.5\%) and email (50.0\%). Three respondents $(100.0 \%)$ indicated other selections writing in farm visits, this would be the same as personal visits (see Table 2). 
Table 2

West Virginia Beef Producers Preferences to Receive Advertisements about Extension Programs

\begin{tabular}{|c|c|c|c|c|c|c|c|c|}
\hline & \multicolumn{4}{|c|}{ Prefer } & \multicolumn{4}{|c|}{ Receive } \\
\hline & \multicolumn{2}{|c|}{ Disagree } & \multicolumn{2}{|c|}{ Agree } & \multicolumn{2}{|c|}{ Disagree } & \multicolumn{2}{|c|}{ Agree } \\
\hline & $\mathrm{N}$ & $\%$ & $\mathrm{~N}$ & $\%$ & $\mathrm{~N}$ & $\%$ & $\mathrm{~N}$ & $\%$ \\
\hline Email & 30 & 33.3 & 60 & 66.7 & 41 & 50.0 & 41 & 50.0 \\
\hline Mail & 2 & 1.6 & 124 & 98.4 & 3 & 2.7 & 109 & 97.3 \\
\hline Phone & 32 & 29.4 & 77 & 70.6 & 40 & 43.5 & 52 & 56.5 \\
\hline $\begin{array}{l}\text { Word of } \\
\text { mouth }\end{array}$ & 27 & 24.1 & 85 & 75.9 & 18 & 17.6 & 84 & 82.4 \\
\hline Newspapers & 18 & 15.8 & 96 & 84.2 & 23 & 22.5 & 79 & 77.5 \\
\hline Newsletters & 4 & 3.3 & 118 & 96.7 & 16 & 15.2 & 89 & 84.8 \\
\hline Radio & 37 & 35.2 & 68 & 64.8 & 45 & 50.6 & 44 & 49.4 \\
\hline Internet & 31 & 33.3 & 62 & 66.7 & 49 & 57.6 & 36 & 42.4 \\
\hline Television & 32 & 31.1 & 71 & 68.9 & 49 & 55.1 & 40 & 44.9 \\
\hline Flyers & 14 & 12.7 & 96 & 87.3 & 21 & 22.1 & 74 & 77.9 \\
\hline $\begin{array}{l}\text { Personal } \\
\text { visits }\end{array}$ & 15 & 13.5 & 96 & 86.5 & 38 & 41.3 & 54 & 58.7 \\
\hline Other & 0 & 0.0 & 3 & 100.0 & 0 & 0.0 & 3 & 100.0 \\
\hline
\end{tabular}

West Virginia beef producers were asked what teaching methods they prefer when attending Extension programs. The top three teaching methods were: demonstrations preferred by $128(100 \%)$ producers, lectures were preferred method of $123(97.7 \%)$ producers, and $54(94.2 \%)$ producers preferred individual consultation. The next three preferred teaching methods were: discussion indicated by $117(93.6 \%)$ 
producers; while, 105 (92.9\%) producers choose fact sheet method; and 108 (91.6\%) producers selected showing DVDs/Videos. Followed by 92 (82.1\%) producers who preferred use of books; 71 (70.3\%) producers indicated they preferred the teaching or demonstration of computer software; and 59 (59.6\%) producers indicated they prefer Internet as a teaching method (see Table 3).

Table 3

Teaching Methods Beef Producers Prefer to Learn

\begin{tabular}{|c|c|c|c|c|c|c|c|c|c|c|c|c|}
\hline & \multicolumn{2}{|c|}{$\begin{array}{l}\text { Strongly } \\
\text { Disagree }\end{array}$} & \multicolumn{2}{|c|}{$\begin{array}{c}\text { Moderately } \\
\text { Disagree }\end{array}$} & \multicolumn{2}{|c|}{ Disagree } & \multicolumn{2}{|c|}{ Agree } & \multicolumn{2}{|c|}{$\begin{array}{c}\text { Moderately } \\
\text { Agree }\end{array}$} & \multicolumn{2}{|c|}{$\begin{array}{c}\text { Strongly } \\
\text { Agree }\end{array}$} \\
\hline & $\mathrm{N}$ & $\%$ & $\mathrm{~N}$ & $\%$ & $\mathrm{~N}$ & $\%$ & $\mathrm{~N}$ & $\%$ & $\mathrm{~N}$ & $\%$ & $\mathrm{~N}$ & $\%$ \\
\hline Demonstration & 0 & .0 & 0 & .0 & 0 & .0 & 32 & 25.0 & 17 & 13.3 & 79 & 61.7 \\
\hline Lecture & 2 & 1.6 & 4 & 3.2 & 2 & 1.6 & 65 & 52.0 & 22 & 17.6 & 30 & 24.0 \\
\hline Discussion & 0 & .0 & 2 & 1.6 & 1 & .8 & 49 & 38.9 & 30 & 23.8 & 44 & 34.9 \\
\hline Internet & 9 & 9.1 & 2 & 2.0 & 29 & 29.3 & 42 & 42.4 & 8 & 8.1 & 9 & 9.1 \\
\hline Fact Sheets & 3 & 2.7 & 1 & .9 & 4 & 3.5 & 50 & 44.2 & 23 & 20.4 & 32 & 28.3 \\
\hline $\begin{array}{l}\text { Showing video- } \\
\text { DVD }\end{array}$ & 4 & 3.4 & 1 & .8 & 5 & 4.2 & 39 & 33.1 & 32 & 27.1 & 37 & 31.4 \\
\hline $\begin{array}{l}\text { teaching \&/or } \\
\text { demonstrating } \\
\text { computer } \\
\text { software }\end{array}$ & 6 & 5.9 & 5 & 5.0 & 19 & 18.8 & 44 & 43.6 & 16 & 15.8 & 11 & 10.9 \\
\hline Books & 4 & 3.6 & 2 & 1.8 & 14 & 12.5 & 58 & 51.8 & 23 & 20.5 & 11 & 9.8 \\
\hline $\begin{array}{l}\text { Individual } \\
\text { consultation }\end{array}$ & 0 & .0 & 0 & .0 & 7 & 5.8 & 48 & 39.7 & 19 & 15.7 & 47 & 38.8 \\
\hline
\end{tabular}

West Virginia beef producers were asked what West Virginia Extension beef programs they were interested. The top four programs were: herd health management as 
indicated by $128(99.2 \%)$ producers, nutrition reported by $122(98.4 \%)$ producers, while 121 (97.6) producers indicated interest in replacement heifer management; and 119 (97.6\%) producers showed interest in forage production \& management. This was followed by 115 (96.6\%) producers who indicated interest in Beef Quality Assurance (BQA) programs; $121(96.1 \%)$ producers reported interest in marketing programs; 114 (95.9\%) producers were interested in record keeping; and 114 (95.8\%) producers also would prefer programs in facility design. One hundred seventeen (95.1\%) producers showed interest in reproduction-fertility management programs; $111(94.9 \%)$ producers indicated an interest in genetic evaluation; 107 (88.5\%) producers indicated interest in the Bull Test; and 108 (81.5\%) producers had an interest in the Livestock Risk Protection (LRP) program (see Table 4).

West Virginia beef producers were ask to indicate how many beef programs they attend per year. Fifty-four (40.9\%) producers indicated they attended one-two programs per year, $38(28.8 \%)$ producers said they attended three-four programs per year, and 11 (8.3\%) said they attend five or more programs a year. Twenty-nine (22.0\%) producers said they did not attend beef Extension programs (see Table 5). 
Table 4

West Virginia Beef Producers Interest in West Virginia Extension Programs

\begin{tabular}{|c|c|c|c|c|c|c|c|c|c|c|c|c|}
\hline & \multicolumn{2}{|c|}{$\begin{array}{l}\text { Strongly } \\
\text { Disagree }\end{array}$} & \multicolumn{2}{|c|}{$\begin{array}{c}\text { Moderately } \\
\text { Disagree }\end{array}$} & \multicolumn{2}{|c|}{ Disagree } & \multicolumn{2}{|c|}{ Agree } & \multicolumn{2}{|c|}{$\begin{array}{c}\text { Moderately } \\
\text { Agree }\end{array}$} & \multicolumn{2}{|c|}{ Strongly Agree } \\
\hline & $\mathrm{N}$ & $\%$ & $\mathrm{~N}$ & $\%$ & $\mathrm{~N}$ & $\%$ & $\mathrm{~N}$ & $\%$ & $\mathrm{~N}$ & $\%$ & $\mathrm{~N}$ & $\%$ \\
\hline Beef Quality Assurance & 1 & .8 & 0 & .0 & 3 & 2.5 & 40 & 33.6 & 17 & 14.3 & 58 & 48.7 \\
\hline Livestock Risk Protection & 1 & .8 & 4 & 3.4 & 5 & 4.2 & 49 & 41.5 & 21 & 17.8 & 38 & 32.2 \\
\hline Replacement heifer mgt. & 0 & .0 & 1 & .8 & 2 & 1.6 & 44 & 35.5 & 26 & 21.0 & 51 & 41.1 \\
\hline Herd health management & 0 & .0 & 1 & .8 & 0 & .0 & 31 & 24.0 & 21 & 16.3 & 76 & 58.9 \\
\hline Reproduction-fertility mgt. & 0 & .0 & 1 & .8 & 5 & 4.1 & 32 & 26.0 & 24 & 19.5 & 61 & 49.6 \\
\hline Facilities design & 0 & .0 & 1 & .8 & 4 & 3.4 & 48 & 40.3 & 24 & 20.2 & 42 & 35.3 \\
\hline Marketing & 0 & .0 & 2 & 1.6 & 3 & 2.4 & 38 & 30.2 & 17 & 13.5 & 66 & 52.4 \\
\hline Record keeping & 2 & 1.7 & 1 & .8 & 2 & 1.7 & 46 & 38.7 & 31 & 26.1 & 37 & 31.1 \\
\hline Nutrition & 0 & .0 & 2 & 1.6 & 0 & .0 & 34 & 27.4 & 25 & 20.2 & 63 & 50.8 \\
\hline Forage production \& Mgt. & 0 & .0 & 0 & .0 & 3 & 2.5 & 30 & 24.6 & 23 & 18.9 & 66 & 54.1 \\
\hline Genetic evaluation & 0 & .0 & 0 & .0 & 6 & 5.1 & 39 & 33.3 & 27 & 23.1 & 45 & 38.5 \\
\hline Bull Test & 1 & .8 & 3 & 2.5 & 10 & 8.3 & 38 & 31.4 & 25 & 20.7 & 44 & 36.4 \\
\hline
\end{tabular}


Table 5

Number of Beef Extension Programs Attended Per Year as Reported by Producers

\begin{tabular}{lll}
\hline & $\mathrm{N}$ & $\%$ \\
\hline 0 & 29 & 22.0 \\
$1-2$ & 54 & 40.9 \\
$3-4$ & 38 & 28.8 \\
5 or more & 11 & 8.3 \\
\hline
\end{tabular}

West Virginia beef producers were asked to indicate what percentage of beef programs reflect their attendance at Extension programming. Sixty-three (48.5\%) respondents selected $0-25 \%, 25(19.2 \%)$ producers selected $25-50$ percent, $25(19.2 \%)$ producers selected 76 percent or more and $17(13.1 \%)$ producers indicated beef programs account for $51-75 \%$ of their Extension programming (see Table 6).

Table 6

Percentage of Extension Programming that are Beef Programs

\begin{tabular}{lcc}
\hline & $\mathrm{N}$ & $\%$ \\
\hline $0-25$ & 63 & 48.5 \\
$26-50$ & 25 & 19.2 \\
$51-75$ & 17 & 13.1 \\
76 or more & 25 & 19.2 \\
\hline
\end{tabular}

West Virginia Beef producers were asked about the size of their herds. Thirty-six (27.1\%) producers indicated their herds were between 1-20 head of cattle; $32(24.1 \%)$ producers indicated their herds were between 21-40 head of cattle. Twenty-seven (20.3\%) producers indicated their herd size was between 41-60 head of cattle; $19(14.3 \%)$ 
producers indicated their herds were made up of over 100 cattle. Thirteen $(9.8 \%)$

producers said their herds were between $61-80$ head; and six (4.5\%) producers said their herds were between 81-100 head (see Table 7).

Table 7

Head of Cattle per Farm

\begin{tabular}{lcc}
\hline & $\mathrm{N}$ & $\%$ \\
\hline $1-20$ & 36 & 27.1 \\
$21-40$ & 32 & 24.1 \\
$41-60$ & 27 & 20.3 \\
$61-80$ & 13 & 9.8 \\
$81-100$ & 6 & 4.5 \\
101 or more & 19 & 14.3 \\
\hline
\end{tabular}

West Virginia beef producers were asked to indicate what type of beef operation they run. One hundred seven $(79.9 \%)$ producers indicated they operated a commercial cow/calf operation selling feeder cattle; 20 (14.9\%) producers indicated they operated a stocker operation and sold as yearlings; $19(14.2 \%)$ producers said they operated a seedstock operation, meaning registered purebred cattle; and $14(10.4 \%)$ producers indicated they operated a commercial cow-calf operation, retaining ownership through slaughter. Seven (5.2\%) producers indicated they had other operations including: butcher for self, club calf production, cow/calf breeding, purebred Angus selling bulls and heifers; and six (4.5\%) producers said they had a feedlot operation (see Table 8). 
Table 8

Types of Beef Cattle Operations

\begin{tabular}{lcc}
\hline & $\mathrm{N}$ & $\%$ \\
\hline Commercial cow-calf selling feeder cattle & 107 & 79.9 \\
$\begin{array}{l}\text { Commercial cow-calf retaining ownership } \\
\text { through slaughter }\end{array}$ & 14 & 10.4 \\
Seedstock & 19 & 14.2 \\
Stocker operations as yearlings & 20 & 14.9 \\
Feedlot & 6 & 4.5 \\
Other & 7 & 5.2 \\
\hline
\end{tabular}

West Virginia beef producers were asked to indicate their age range. Sixty-four (49.2\%) producers indicated they were 60 years or older; 27 (20.8\%) producers were 5059 years of age, $22(16.9 \%)$ producers indicated they were between $40-49$ years of age; $10(7.7 \%)$ producers said they were between 30-39 years old while four (3.1\%) producers indicated they were between 20-29 years old; and three $(2.3 \%)$ producers said they were younger than 20 (see Table 9).

When asked how many years their operation had been in business, $92(70.2 \%)$ producers indicated they had been in business for over 25 years. Eleven $(8.4 \%)$ producers had been in business for 21-25 years; $11(8.4 \%)$ producers had been in business 16-20 years; eight (6.1\%) producers had been in business for 11-15 years; six (4.6\%) producers indicated 6-10 years; and three producers said they had been in business 1-5 years (see Table 10). 
Table 9

Beef Producers Age Range

\begin{tabular}{lcc}
\hline & $\mathrm{N}$ & $\%$ \\
\hline Younger than 20 years & 3 & 2.3 \\
$20-29$ & 4 & 3.1 \\
$30-39$ & 10 & 7.7 \\
$40-49$ & 22 & 16.9 \\
$50-59$ & 27 & 20.8 \\
60 or older & 64 & 49.2 \\
\hline
\end{tabular}

Table 10

Years of Operation

\begin{tabular}{lcc}
\hline & $\mathrm{N}$ & $\%$ \\
\hline $1-5$ years & 3 & 2.3 \\
$6-10$ years & 6 & 4.6 \\
$11-15$ years & 8 & 6.1 \\
$16-20$ years & 11 & 8.4 \\
$21-25$ years & 11 & 8.4 \\
26 or more & 92 & 70.2 \\
\hline
\end{tabular}

West Virginia beef producers were asked to indicate how their local Extension Agent handles beef programming. Sixty (45.8\%) producers said their Extension Agent has responsibility for their county only and 30 (22.9\%) producers said their Extension 
Agent has responsibility for two or more counties. Forty-one (31.3\%) producers were not sure (see Table 11).

Table 11

Local Extension Agent, who Handles Beef Programming

\begin{tabular}{lll}
\hline & $\mathrm{N}$ & $\%$ \\
\hline Has responsibility for my county only & 60 & 45.8 \\
Has responsibility for two or more counties & 30 & 22.9 \\
I am not sure & 41 & 31.3 \\
\hline
\end{tabular}

West Virginia beef producers were asked to rank six sources in which they might go to for beef related questions. The responses were then recoded to create a summed score for each source. The scores were then used to rank the information sources. According to West Virginia beef producers the Extension Agent is the most popular source to use when information is needed, followed by: veterinarians, neighbors, Extension Specialist, farm store employees, and other (see Table 12). The other sources included: family members, the Internet, books, ag teachers, and publications, etc.

The data were analyzed to determine the most popular choice by beef producers of where they would go to get answers to their questions. The most popular sources were, WVU Extension Agents, followed by veterinarians, Extension Specialist, neighbors, farm store employees, and then other sources (see Table 12). 
Table 12

How do Beef Producers get Answers to Their Beef Questions?

\begin{tabular}{lccc}
\hline & Mean & Std Deviation & Sum \\
\hline WVU Extension Agent & 4.72 & 1.40 & 415 \\
Veterinarian & 4.64 & 1.25 & 357 \\
Neighbor & 4.03 & 1.44 & 294 \\
Extension Specialist & 4.15 & 1.50 & 282 \\
Farm Store Employee & 3.51 & 1.38 & 228 \\
Other & 5.07 & 1.44 & 142 \\
\hline
\end{tabular}

\section{Extension Agents}

Extension Agents were asked to evaluate themselves and their Extension programs related to beef production. Thirty-one (83.8\%) agents were in some level of agreement that they offered Extension programs related to beef production. Twenty-three (62.1\%) agents strongly agreed, four (10.8) agents moderately agreed, and four $(10.8 \%)$ agents agreed they offered Extension programs relating to beef. Six (16.2\%) agents were in some level of disagreement that they offered Extension programs related to beef production. Five (13.5\%) agents strongly disagreed, and one (2.7\%) agent moderately disagreed that they offer Extension programs related to beef production (see Table 13).

West Virginia University Extension Agents were asked if they could answer most questions about beef production and $31(86.1 \%)$ agents were in some form of agreement. Thirteen (36.1\%) agents strongly agreed, 10 (27.8\%) agents moderately agreed, and eight $(22.2 \%)$ agents agreed that they could answer most questions related to beef production. Five (14\%) agents were in some form of disagreement that they could answer most beef 
questions. Two (5.6\%) agents strongly disagreed, two (5.6\%) agents moderately disagreed, and one (2.8\%) agent disagreed in being able to answer most questions about beef production (see Table 13).

When asked if West Virginia University Extension Agents return beef producers phone calls in a timely manner, 37 (100\%) were in some form of agreement. Twentyseven (73.0\%) strongly agreed, five (13.5\%) agents moderately agreed, and five $(13.5 \%)$ agents agreed that they return beef producers phone calls in a timely manner (see Table 13).

West Virginia University Extension Agents were asked if they returned beef producers' emails in a timely manner, Thirty-eight (100\%) Extension Agents were in some form of agreement. Twenty-eight (80.0\%) agents strongly agreed, four $(11.4 \%)$ agents agreed, and three (8.6\%) agents moderately agreed in returning beef producer's emails in a timely manner (see Table 13).

When ask if beef production programs offered in their counties were well attended, $28(82.3 \%)$ agents were in some form of agreement. Ten (29.4\%) agents strongly agreed, 10 (29.4\%) agents moderately agreed, and eight (23.5\%) agents agreed that beef production programs were well attended. Six (17.6\%) agents were in some form of disagreement, three $(8.8 \%)$ agents strongly disagree, two $(5.9 \%)$ agents disagree, and one $(2.9 \%)$ agent moderately disagree that their beef production programs were well attended (see Table 13).

West Virginia University Extension Agents were asked if beef production practices taught were readily accepted. Twelve (37.5\%) agents agreed, eight $(25.0 \%)$ agents moderately agreed, and four (12.5\%) agents strongly agreed that beef production 
practices taught were being readily accepted. Six (18.8\%) agents disagreed, one (3.1\%) agent moderately disagreed, and one agent (3.1\%) strongly disagreed that beef production practices being taught were being readily accepted (see Table 13).

When asked if West Virginia University Extension Agents consider beef production one of their specialty areas, twenty-one (55.3\%) agents were in some form of agreement. Twelve (31.6\%) agents strongly agreed, six (15.8\%) agents agreed, and three (7.9\%) agents moderately agreed that they consider beef production as one of their specialty areas. Seven (18.4\%) agents strongly disagreed, six (15.8\%) agents disagreed, and four $(10.5 \%)$ agents moderately disagreed that beef production was one of their specialty areas (see Table 13).

West Virginia University Extension Agents were asked what methods of advertisement they use to promote beef programs. The top four methods of advertising reported were: word of mouth (97.1\%), newspapers (97.0\%), mail (94.3\%), and newsletters (81.9\%). This was followed by personal visits $(81.8 \%)$, phone $(79.9 \%)$, flyers $(72.7 \%)$, and other sources $(50.0 \%)$. The least reported methods of advertising Extension Agents prefer to use were: email (49.9\%), Internet (45.5\%), radio (36.4\%), and television (27.3\%). The other sources were magazines (see Table 14). 
Table 13

Self Evaluation of West Virginia University Extension Agents and Their Beef Programs

\begin{tabular}{|c|c|c|c|c|c|c|c|c|c|c|c|c|}
\hline & \multicolumn{2}{|c|}{$\begin{array}{l}\text { Strongly } \\
\text { Disagree }\end{array}$} & \multicolumn{2}{|c|}{$\begin{array}{l}\text { Moderately } \\
\text { Disagree }\end{array}$} & \multicolumn{2}{|c|}{ Disagree } & \multicolumn{2}{|c|}{ Agree } & \multicolumn{2}{|c|}{$\begin{array}{l}\text { Moderately } \\
\text { Agree }\end{array}$} & \multicolumn{2}{|c|}{ Strongly Agree } \\
\hline & $\mathrm{N}$ & $\%$ & $\mathrm{~N}$ & $\%$ & $\mathrm{~N}$ & $\%$ & $\mathrm{~N}$ & $\%$ & $\mathrm{~N}$ & $\%$ & $\mathrm{~N}$ & $\%$ \\
\hline $\begin{array}{l}\text { Offer programs in beef } \\
\text { production }\end{array}$ & 5 & 13.5 & 1 & 2.7 & 0 & 0.0 & 4 & 10.8 & 4 & 10.8 & 23 & 62.2 \\
\hline $\begin{array}{l}\text { Able to answer most } \\
\text { questions about beef } \\
\text { production }\end{array}$ & 2 & 5.6 & 2 & 5.6 & 1 & 2.8 & 8 & 22.2 & 10 & 27.8 & 13 & 36.1 \\
\hline $\begin{array}{l}\text { Returns calls in a timely } \\
\text { manner }\end{array}$ & 0 & 0.0 & 0 & 0.0 & 0 & 0.0 & 5 & 13.5 & 5 & 13.5 & 27 & 73.0 \\
\hline $\begin{array}{l}\text { Returns emails in a timely } \\
\text { manner }\end{array}$ & 0 & 0.0 & 0 & 0.0 & 0 & 0.0 & 4 & 11.4 & 3 & 8.6 & 28 & 80.0 \\
\hline $\begin{array}{l}\text { Beef programs are well } \\
\text { attended }\end{array}$ & 3 & 8.8 & 1 & 2.9 & 2 & 5.9 & 8 & 23.5 & 10 & 29.4 & 10 & 29.4 \\
\hline $\begin{array}{l}\text { Beef production practices } \\
\text { are readily accepted }\end{array}$ & 1 & 3.1 & 1 & 3.1 & 6 & 18.8 & 12 & 37.5 & 8 & 25.0 & 4 & 12.5 \\
\hline $\begin{array}{l}\text { Consider beef one of my } \\
\text { specialty areas }\end{array}$ & 7 & 18.4 & 4 & 10.5 & 6 & 15.8 & 6 & 15.8 & 3 & 7.9 & 12 & 31.6 \\
\hline
\end{tabular}


Table 14

Methods of Advertisement West Virginia University Extension Agents Use

\begin{tabular}{|c|c|c|c|c|c|c|c|c|c|c|c|c|}
\hline & \multicolumn{2}{|c|}{$\begin{array}{l}\text { Strongly } \\
\text { Disagree }\end{array}$} & \multicolumn{2}{|c|}{$\begin{array}{l}\text { Moderately } \\
\text { Disagree }\end{array}$} & \multicolumn{2}{|c|}{ Disagree } & \multicolumn{2}{|c|}{ Agree } & \multicolumn{2}{|c|}{$\begin{array}{l}\text { Moderately } \\
\text { Agree }\end{array}$} & \multicolumn{2}{|c|}{$\begin{array}{c}\text { Strongly } \\
\text { Agree }\end{array}$} \\
\hline & $\mathrm{N}$ & $\%$ & $\mathrm{~N}$ & $\%$ & $\mathrm{~N}$ & $\%$ & $\mathrm{~N}$ & $\%$ & $\mathrm{~N}$ & $\%$ & $\mathrm{~N}$ & $\%$ \\
\hline Email & 5 & 14.7 & 2 & 5.9 & 10 & 29.4 & 6 & 17.6 & 5 & 14.7 & 6 & 17.6 \\
\hline Mail & 2 & 5.7 & 0 & 0.0 & 0 & 0.0 & 6 & 17.1 & 3 & 8.6 & 24 & 68.6 \\
\hline Phone & 3 & 8.6 & 1 & 2.9 & 3 & 8.6 & 13 & 37.1 & 4 & 11.4 & 11 & 31.4 \\
\hline $\begin{array}{l}\text { Word of } \\
\text { mouth }\end{array}$ & 1 & 2.9 & 0 & 0.0 & 0 & 0.0 & 13 & 37.1 & 6 & 17.1 & 15 & 42.9 \\
\hline Newspapers & 0 & 0.0 & 0 & 0.0 & 1 & 2.9 & 8 & 23.5 & 7 & 20.6 & 18 & 52.9 \\
\hline Newsletters & 4 & 12.1 & 0 & 0.0 & 2 & 6.1 & 5 & 15.2 & 7 & 21.2 & 15 & 45.5 \\
\hline Radio & 8 & 24.2 & 1 & 3.0 & 12 & 36.4 & 5 & 15.2 & 3 & 9.1 & 4 & 12.1 \\
\hline Internet & 5 & 15.2 & 4 & 12.1 & 9 & 27.3 & 11 & 33.3 & 1 & 3.0 & 3 & 9.1 \\
\hline Television & 12 & 36.4 & 1 & 3.0 & 11 & 33.3 & 6 & 18.2 & 1 & 3.0 & 2 & 6.1 \\
\hline Flyers & 4 & 12.1 & 1 & 3.0 & 4 & 12.1 & 8 & 24.2 & 9 & 27.3 & 7 & 21.2 \\
\hline Personal visits & 3 & 9.1 & 0 & 0.0 & 3 & 9.1 & 10 & 30.3 & 9 & 27.3 & 8 & 24.2 \\
\hline Other & 0 & 0.0 & 0 & 0.0 & 0 & 0.0 & 0 & 0.0 & 1 & 50.0 & 1 & 50.0 \\
\hline
\end{tabular}

West Virginia University Extension Agents were asked what teaching methods they prefer to use in beef programs. The top teaching methods were: discussion $(94.1 \%)$, fact sheets (91.2\%), and individual consultation (91.0\%), followed by: demonstration $(88.3 \%)$, lecture $(88.2 \%)$, and showing video or DVD (85.4\%). Extension Agents in West Virginia prefer not to use the following methods: books $(66.7 \%)$, Internet $(61.8 \%)$, 
and teaching or demonstrating computer software (33.3\%)(see Table 15). Other sources Extension Agents may use include: Power Point, one-on-one, farm days, and field days. Table 15

Teaching Methods Extension Agents Prefer to Use to Deliver Beef Programs

\begin{tabular}{|c|c|c|c|c|c|c|c|c|c|c|c|c|}
\hline & \multicolumn{2}{|c|}{$\begin{array}{l}\text { Strongly } \\
\text { Disagree }\end{array}$} & \multicolumn{2}{|c|}{$\begin{array}{c}\text { Moderately } \\
\text { Disagree }\end{array}$} & \multicolumn{2}{|c|}{ Disagree } & \multicolumn{2}{|c|}{ Agree } & \multicolumn{2}{|c|}{$\begin{array}{c}\text { Moderately } \\
\text { Agree }\end{array}$} & \multicolumn{2}{|c|}{$\begin{array}{l}\text { Strongly } \\
\text { Agree }\end{array}$} \\
\hline & $\mathrm{N}$ & $\%$ & $\mathrm{~N}$ & $\%$ & $\mathrm{~N}$ & $\%$ & $\mathrm{~N}$ & $\%$ & $\mathrm{~N}$ & $\%$ & $\mathrm{~N}$ & $\%$ \\
\hline Demonstration & 3 & 8.8 & 0 & 0.0 & 1 & 2.9 & 11 & 32.4 & 5 & 14.7 & 14 & 41.2 \\
\hline Lecture & 2 & 5.9 & 2 & 5.9 & 0 & 0.0 & 8 & 23.5 & 5 & 14.7 & 17 & 50.0 \\
\hline Discussion & 2 & 5.9 & 0 & 0.0 & 0 & 0.0 & 8 & 23.5 & 7 & 20.6 & 17 & 50.0 \\
\hline Internet & 8 & 23.5 & 3 & 8.8 & 10 & 29.4 & 10 & 29.4 & 1 & 2.9 & 2 & 5.9 \\
\hline Fact Sheets & 1 & 2.9 & 0 & 0.0 & 2 & 5.9 & 7 & 20.6 & 13 & 38.2 & 11 & 32.4 \\
\hline $\begin{array}{l}\text { Showing video- } \\
\text { DVD }\end{array}$ & 2 & 5.9 & 1 & 2.9 & 2 & 5.9 & 16 & 47.1 & 9 & 26.5 & 4 & 11.8 \\
\hline $\begin{array}{l}\text { Teaching and-or } \\
\text { demonstrating } \\
\text { computer software }\end{array}$ & 9 & 27.3 & 2 & 6.1 & 11 & 33.3 & 8 & 24.2 & 2 & 6.1 & 1 & 3.0 \\
\hline Books & 8 & 24.2 & 2 & 6.1 & 8 & 24.2 & 10 & 30.3 & 2 & 6.1 & 3 & 9.1 \\
\hline $\begin{array}{l}\text { Individual } \\
\text { consultation }\end{array}$ & 2 & 6.1 & 1 & 3.0 & 0 & .0 & 5 & 15.2 & 5 & 15.2 & 20 & 60.6 \\
\hline Other & 0 & .0 & 0 & .0 & 0 & .0 & 1 & 20.0 & 0 & .0 & 4 & 80.0 \\
\hline
\end{tabular}

West Virginia University Extension Agents were ask what beef programs they offered in their counties. The top programs offered by Extension Agents were: nutrition $(90.9 \%)$, forage production \& management $(90.9 \%)$, reproduction-fertility management, and marketing (87.9\%) (see Table 16). This was followed by Beef Quality Assurance 
Table 16

Beef Programs Offered by West Virginia University Extension Agents

\begin{tabular}{|c|c|c|c|c|c|c|c|c|c|c|c|c|}
\hline & \multicolumn{2}{|c|}{$\begin{array}{l}\text { Strongly } \\
\text { Disagree }\end{array}$} & \multicolumn{2}{|c|}{$\begin{array}{c}\text { Moderately } \\
\text { Disagree }\end{array}$} & \multicolumn{2}{|c|}{ Disagree } & \multicolumn{2}{|c|}{ Agree } & \multicolumn{2}{|c|}{$\begin{array}{c}\text { Moderately } \\
\text { Agree }\end{array}$} & \multicolumn{2}{|c|}{$\begin{array}{l}\text { Strongly } \\
\text { Agree }\end{array}$} \\
\hline & $\mathrm{N}$ & $\%$ & $\mathrm{~N}$ & $\%$ & $\mathrm{~N}$ & $\%$ & $\mathrm{~N}$ & $\%$ & $\mathrm{~N}$ & $\%$ & $\mathrm{~N}$ & $\%$ \\
\hline $\begin{array}{l}\text { Beef Quality } \\
\text { Assurance } \\
\text { (BQA) }\end{array}$ & 2 & 6.3 & 0 & 0.0 & 2 & 6.3 & 4 & 12.5 & 8 & 25.0 & 16 & 50.0 \\
\hline $\begin{array}{l}\text { Livestock } \\
\text { Risk } \\
\text { Protection } \\
\text { (LRP) }\end{array}$ & 2 & 6.3 & 0 & 0.0 & 8 & 25.0 & 11 & 34.4 & 5 & 15.6 & 6 & 18.8 \\
\hline $\begin{array}{l}\text { Replacement } \\
\text { heifer } \\
\text { management }\end{array}$ & 2 & 6.3 & 1 & 3.1 & 6 & 18.8 & 8 & 25.0 & 12 & 37.5 & 3 & 9.4 \\
\hline $\begin{array}{l}\text { Herd health } \\
\text { management }\end{array}$ & 2 & 6.3 & 0 & .0 & 1 & 3.1 & 5 & 15.6 & 5 & 15.6 & 19 & 59.4 \\
\hline Reproduction & 3 & 9.4 & 1 & 3.1 & 2 & 6.3 & 7 & 21.9 & 13 & 40.6 & 6 & 18.8 \\
\hline $\begin{array}{l}\text { Facilities } \\
\text { design }\end{array}$ & 3 & 9.4 & 2 & 6.3 & 3 & 9.4 & 16 & 50.0 & 3 & 9.4 & 5 & 15.6 \\
\hline Marketing & 2 & 6.1 & 0 & 0.0 & 2 & 6.1 & 8 & 24.2 & 4 & 12.1 & 17 & 51.5 \\
\hline $\begin{array}{l}\text { Record } \\
\text { keeping }\end{array}$ & 2 & 6.3 & 1 & 3.1 & 2 & 6.3 & 12 & 37.5 & 9 & 28.1 & 6 & 18.8 \\
\hline Nutrition & 2 & 6.1 & 0 & 0.0 & 1 & 3.0 & 8 & 24.2 & 17 & 51.5 & 5 & 15.2 \\
\hline $\begin{array}{l}\text { Forage } \\
\text { production \& } \\
\text { management }\end{array}$ & 2 & 6.1 & 0 & 0.0 & 1 & 3.0 & 4 & 12.1 & 8 & 24.2 & 18 & 54.5 \\
\hline $\begin{array}{l}\text { Genetic } \\
\text { evaluation }\end{array}$ & 2 & 6.3 & 2 & 6.3 & 3 & 9.4 & 11 & 34.4 & 5 & 15.6 & 9 & 28.1 \\
\hline Bull Test & 1 & 3.1 & 0 & 0.0 & 5 & 15.6 & 10 & 31.3 & 2 & 6.3 & 14 & 43.8 \\
\hline
\end{tabular}


(87.5\%), record keeping (84.4\%), Bull Test (81.3\%), and genetic evaluation $(78.1 \%)$.

Extension Agents also offer: facility design (75.0\%), replacement heifer production, and Livestock Risk Protection (68.8\%) (see Table 16).

When asked, how many beef programs they offer a year, eighteen (47.4\%) agents said they offered 5 or more programs, eight (21.1\%) agents offered 3-4 beef programs, and five (13.2\%) agents offered 1-2 beef programs a year. Seven (18.4\%) agents said they did not offer any beef programs (see Table 17).

Table 17

Number of Beef Extension Programs, Agents Offer per Year

\begin{tabular}{lcc}
\hline & $\mathrm{N}$ & $\%$ \\
\hline None & 7 & 18.4 \\
$1-2$ & 5 & 13.2 \\
$3-4$ & 8 & 21.1 \\
5 or more & 18 & 47.4 \\
\hline
\end{tabular}

West Virginia Extension Agents were asked what percentage of your programs offered per year is beef related. Twenty-one (55.3\%) indicated 0-25\%, $16(42.1 \%)$, agents indicated $26-50 \%$, and one (2.6\%) agent indicated that $51-75$ percent of their programs are beef related (see Table 18).

When asked how many beef producers they work with each year, $11(28.9 \%)$ agents indicated they worked with over 100 producers each year, eight $(21.1 \%)$ agents indicated they worked with 0 -10 per year, five (13.2\%) agents said they worked with 4150 per year, three $(7.9 \%)$ agents each worked with $11-20$, and $20-30$ producers. Two (5.3\%) agents each said they worked in the follow categories: $31-40,71-80$, and $81-90$ 
producers per year. One (2.6\%) agent worked with 51-60 producers each years and another agent (2.6\%) works with 61-70 producers per year (see Table 19).

Table 18

Average Attendance of Extension Programs per Year

\begin{tabular}{lcc}
\hline & $\mathrm{N}$ & $\%$ \\
\hline $0-25$ & 21 & 55.3 \\
$26-50$ & 16 & 42.1 \\
$51-75$ & 1 & 2.6 \\
\hline
\end{tabular}

When asked about the average beef herd size in their county, $19(54.3 \%)$ agents indicated 21-40 head. Nine (25.7\%) Extension Agents indicated 1-20 head. Three (8.6\%) agents indicated 41-60 head. Two (5.7\%) agents indicated 61-80 head, and two (5.7\%) agents indicated 81-100 head (see Table 20).

When Extension Agents were asked what best describes their county's beef operations, 33 (89.2\%) agents indicated commercial cow/operation selling feeder cattle; $11(28.9 \%)$ agents indicated stocker operations, selling as yearlings; $10(26.3 \%)$ agents indicated commercial cow/calf operations, retaining ownership through slaughter; nine (23.7\%) agents indicated seedstock, purebred operations; six (15.8\%) agents indicated feedlot operations; and two (5.3\%) agents indicated other operations. The other operations were: hobby farm and grass fed beef operations (see Table 21). 
Table 19

Average Number of West Virginia Beef Producers Worked with Each Year

\begin{tabular}{lcc}
\hline & $\mathrm{N}$ & $\%$ \\
\hline $0-10$ & 8 & 21.1 \\
$11-20$ & 3 & 7.9 \\
$21-30$ & 3 & 7.9 \\
$31-40$ & 2 & 5.3 \\
$41-50$ & 5 & 13.2 \\
$51-60$ & 1 & 2.6 \\
$61-70$ & 1 & 2.6 \\
$71-80$ & 2 & 5.3 \\
$81-90$ & 2 & 5.3 \\
101 or more & 11 & 28.9 \\
\hline
\end{tabular}

Table 20

Average Herd Size per Farm

\begin{tabular}{lcc}
\hline & $\mathrm{N}$ & $\%$ \\
\hline $1-20$ & 9 & 25.7 \\
$21-40$ & 19 & 54.3 \\
$41-60$ & 3 & 8.6 \\
$61-80$ & 2 & 5.7 \\
$81-100$ & 2 & 5.7 \\
\hline
\end{tabular}


Table 21

Types of Beef Operations in Their County

\begin{tabular}{lcc}
\hline & $\mathrm{N}$ & $\%$ \\
\hline Commercial cow-calf selling feeder cattle & 33 & 89.2 \\
$\begin{array}{l}\text { Commercial cow-calf retaining ownership } \\
\text { through slaughter }\end{array}$ & 10 & 26.3 \\
Seedstock & 9 & 23.7 \\
Stocker operations as yearlings & 11 & 28.9 \\
Feedlot & 6 & 15.8 \\
Other & 2 & 5.3 \\
\hline
\end{tabular}

When Extension Agents were asked about the average age of farmers in their county, $19(51.4 \%)$ agents indicated 50-59 years of age. Thirteen (35.1\%) agents indicated 60 or older, and five (13.5\%) agents indicated $40-49$ years of age (see Table 22).

Table 22

Average Age of Farmers in Their County

\begin{tabular}{lcc}
\hline & $\mathrm{N}$ & $\%$ \\
\hline $40-49$ & 5 & 13.5 \\
$50-59$ & 19 & 51.4 \\
60 or older & 13 & 35.1 \\
\hline
\end{tabular}

Extension Agents were asked what the average years of operation for their beef producers in their county. Seventeen (48.6\%) agents indicated over 25 years, $11(31.4 \%)$ 
agents indicated $21-25$ years, five (14.3\%) agents indicated $16-20$ years, one (2.9\%) agent indicated $11-15$ years, and one (2.9\%) agent indicated less than a year of operation (see Table 23).

Table 23

Average Years of Beef Cattle Operation in Their County

\begin{tabular}{lcc}
\hline & $\mathrm{N}$ & $\%$ \\
\hline Less than 1 year & 1 & 2.9 \\
$11-15$ years & 1 & 2.9 \\
$16-20$ years & 5 & 14.3 \\
$21-25$ years & 11 & 31.4 \\
26 or more years & 17 & 48.6 \\
\hline
\end{tabular}

West Virginia Extension Agents were asked to rank six sources beef producers might go to for answers to beef related questions. The responses were then recoded to create a summed score for each source. The scores were then used to rank the information sources. According to Extension Agents in West Virginia neighbors were the most popular source for beef producers to use when a beef question needed answered. The other sources in order were, WVU Extension Agents, farm store employee, veterinarian, Extension Specialist, and other sources (see Table 24). 
Table 24

Beef Produces Sources of Information

\begin{tabular}{lc}
\hline & Sum \\
\hline Neighbor & 166 \\
WVU Extension Agent & 158 \\
Farm Store Employee & 132 \\
Veterinarian & 123 \\
Extension Specialist & 101 \\
Other & 45 \\
\hline
\end{tabular}

\section{Comparisons of Extension Agents and West Virginia Beef producers}

A $t$-test statistical procedure was used to determine if statistical differences existed in the means of the two groups Extension Agents and West Virginia beef producers on the use of the WVU Extension Agent as a source to get answers.

\section{The WVU Extension Agent}

The null hypothesis $\mathrm{H}_{0}=M_{\text {Extension }}=M_{\text {producer, }}$ was tested. The alternative hypothesis was $\mathrm{H}_{1}=M_{\text {Extension }} \neq M_{\text {producer. }}$ The mean average of group 1 was 4.51 with a standard deviation of 1.22 . The mean average of group 2 was 4.72 with a standard deviation of 1.40 (see Table 25).

An independent $t$-test statistical analysis procedure was used to compare the means of group 1 and group 2. The statistical analysis results $(t=-0.747, \mathrm{df}=121)$ were not significant at $\alpha \leq .05$. The researcher failed to reject the null there was no difference between the groups with regards of using the Extension Agent as a source of information. 
Table 25

Comparison of the Mean Scores of the WVU Extension Agent as a Source of Information

\begin{tabular}{lccccc}
\hline & $\mathrm{N}$ & $M$ & $S D$ & $d f$ & $t$ \\
\hline Extension & 35 & 4.51 & 1.22 & 121 & -0.747 \\
Producers & 89 & 4.72 & 1.40 & & \\
\hline
\end{tabular}

$* \alpha \leq .05$

\section{The Veterinarian}

A $t$-test statistical procedure was used to determine if statistical differences existed in the means of the two groups Extension Agents and West Virginia beef producers on the use of a veterinarian as a source to get answers to beef related questions.

The null hypothesis $\mathrm{H}_{0}=M_{\text {Extension }}=M_{\text {producer, }}$ was tested. The alternative hypothesis was $\mathrm{H}_{1}=M_{\text {Extension }} \neq M_{\text {producer }}$. The mean average of group 1 was 3.42 with a standard deviation of 1.34. The mean average of group 2 was 4.64 with a standard deviation of 1.25 (see Table 26).

An independent T-test statistical analysis procedure was used to compare the means of group 1 and group 2. The statistical analysis results $(t=-4.736, \mathrm{df}=111)$ were significant at $\alpha \leq .05$. The null hypothesis was rejected and the alternative hypothesis, $\mathrm{H}_{1}=M_{\text {Extension }} \neq M_{\text {producer, }}$, was accepted. There was a difference between Beef producers and Extension Agents. The difference between the means of group1 and group 2 exhibited a medium effect size (Cohen, 1998). 
Table 26

Comparison of Mean Scores of Veterinarians as a Source of Information

\begin{tabular}{llllll}
\hline & $\mathrm{N}$ & $M$ & $S D$ & $d f$ & $t$ \\
\hline Extension & 36 & 3.42 & 1.43 & 111 & $-4.736^{*}$ \\
Producers & 79 & 4.64 & 1.35 & & \\
\hline$* a \leq .05$ & & &
\end{tabular}

$*_{\alpha} \leq .05$

\section{The Neighbor}

A $t$-test statistical procedure was used to determine if statistical differences existed in the means of the two groups Extension Agents and West Virginia beef producers on the use of a neighbor as a source to get answers to beef related questions.

The null hypothesis $\mathrm{H}_{0}=M_{\text {Extension }}=M_{\text {producer, }}$ was tested. The alternative hypothesis was $\mathrm{H}_{1}=M_{\text {Extension }} \neq M_{\text {producer }}$. The mean average of group 1 was 4.88 with a standard deviation of 1.34 . The mean average of group 2 was 4.03 with a standard deviation of 1.44 (see Table 27).

An independent $t$-test statistical analysis procedure was used to compare the means of group 1 and group 2 . The statistical analysis results $(t=2.915, \mathrm{df}=105)$ were significant at $\alpha \leq .05$. The null hypothesis was rejected and the alternative hypothesis, $\mathrm{H}_{1}=M_{\text {Extension }} \neq M_{\text {producer, }}$, was accepted. There was a difference between Beef producers and Extension Agents. The difference between the means of group1 and group 2 exhibited a large effect size (Cohen, 1998). 
Table 27

Comparison of the Mean Scores of Neighbor as a Source of Information

\begin{tabular}{lccccc}
\hline & $\mathrm{N}$ & $M$ & $S D$ & $d f$ & $t$ \\
\hline Extension & 34 & 4.88 & 1.34 & 105 & $2.915^{*}$ \\
Producers & 75 & 4.03 & 1.44 & & \\
\hline
\end{tabular}

$*_{\alpha} \leq .05$

\section{The Extension Specialist}

A $t$-test statistical procedure was used to determine if statistical differences existed in the means of the two groups Extension Agents and West Virginia beef producers on the use of a WVU Extension Specialist as a source to get answers to beef related questions.

The null hypothesis $\mathrm{H}_{0}=M_{\text {Extension }}=M_{\text {producer, }}$ was tested. The alternative hypothesis was $\mathrm{H}_{1}=M_{\text {Extension }} \neq M_{\text {producer }}$. The mean average of group 1 was 2.97 with a standard deviation of 1.36 . The mean average of group 2 was 4.15 with a standard deviation of 1.50 (see Table 28).

An independent $t$-test statistical analysis procedure was used to compare the means of group 1 and group 2. The statistical analysis results $(t=-3.851, \mathrm{df}=100)$ were significant at $\alpha \leq .05$. The null hypothesis was rejected and the alternative hypothesis, $\mathrm{H}_{1}=M_{\text {Extension }} \neq M_{\text {producer, }}$, was accepted. There was a difference between Beef producers and Extension Agents. The difference between the means of group1 and group 2 exhibited a medium (Cohen, 1998). 
Table 28

Comparison of Extension Specialist as a Source of Information

\begin{tabular}{lccccc}
\hline & $\mathrm{N}$ & $M$ & $S D$ & $d f$ & $t$ \\
\hline Extension & 34 & 2.97 & 1.36 & 100 & $-3.851^{*}$ \\
Producers & 75 & 4.15 & 1.50 & & \\
\hline
\end{tabular}

$* \alpha \leq .05$

\section{The Farm Store Employee}

A $t$-test statistical procedure was used to determine if statistical differences existed in the means of the two groups Extension Agents and West Virginia beef producers on the use of a farm store employee as a source to get answers to beef related questions.

The null hypothesis $\mathrm{H}_{0}=M_{\text {Extension }}=M_{\text {producer, }}$ was tested. The alternative hypothesis was $\mathrm{H}_{1}=M_{\text {Extension }} \neq M_{\text {producer }}$. The mean average of group 1 was 4.00 with a standard deviation of 1.39. The mean average of group 2 was 3.51 with a standard deviation of 1.38 (see Table 29).

An independent $t$-test statistical analysis procedure was used to compare the means of group 1 and group 2. The statistical analysis results $(t=1.663, \mathrm{df}=96)$ were not significant at $\alpha \leq .05$. The researcher failed to reject the null and did not accept the alternative hypothesis. There was no difference between the groups. 
Table 29

The Farm Store Employee as a Source of Information

\begin{tabular}{llllll}
\hline & $\mathrm{N}$ & $M$ & $S D$ & $d f$ & $t$ \\
\hline Extension & 33 & 4.00 & 1.39 & 96 & 1.663 \\
Producers & 66 & 3.51 & 1.38 & & \\
\hline
\end{tabular}

$* \alpha \leq .05$

\section{The Other Sources of Information}

A $t$-test statistical procedure was used to determine if statistical differences existed in the means of the two groups Extension Agents and West Virginia beef producers on the use of other sources of information to get answers to beef related questions.

The null hypothesis $\mathrm{H}_{0}=M_{\text {Extension }}=M_{\text {producer, was tested. The alternative }}$ hypothesis was $\mathrm{H}_{1}=M_{\text {Extension }} \neq M_{\text {producer }}$. The mean average of group 1 was 3.46 with a standard deviation of 2.18. The mean average of group 2 was 5.07 with a standard deviation of 1.44 (see Table 30).

An independent $t$-test statistical analysis procedure was used to compare the means of group 1 and group 2. The statistical analysis results $(t=-2.425, \mathrm{df}=17)$ were significant at $\alpha \leq .05$. The null hypothesis was rejected and the alternative hypothesis, $\mathrm{H}_{1}=M_{\text {Extension }} \neq M_{\text {producer, }}$, was accepted. There was a difference between Beef producers and Extension Agents. The difference between he means of group1 and group 2 exhibited a medium effect size (Cohen, 1998). 
Table 30

Comparison of Mean Scores of Other Sources of Information

\begin{tabular}{llllll}
\hline & $\mathrm{N}$ & $M$ & $S D$ & $d f$ & $t$ \\
\hline Extension & 14 & 3.46 & 2.18 & 17 & -2.425 \\
Producers & 28 & 5.07 & 1.44 & & \\
\hline
\end{tabular}

$* \alpha \leq .05$

West Virginia beef producers were asked how they prefer to learn about Extension programs, while Extension Agents were asked what methods of advertising programs they use. Sixty $(66.7 \%)$ beef producers indicated they would like to receive information about programming by email. Extension Agents were divided on the issue with 17 (50.0\%) agents who agreed and 17 (50.0\%) agents who disagreed on email (see Table 31).

Mail was another means of advertisement that $124(98.4 \%)$ beef producers indicated they would prefer, while 33 (94.3\%) Extension Agents indicated they used mail as a source of advertisement. Another source of information was the telephone. Twentyeight (80.0\%) Extension Agents indicated they use telephone, where only 77 (70.7\%) beef producers indicated they would prefer to use the telephone as a method of advertisement (see Table 31).

When asked if newspapers was a preferred sources of advertisement, 96 (84.2\%) beef producers agreed, while as 33 (97.1\%) Extension agents agreed they used the newspaper to advertise programs. Twenty-seven (81.8\%) Extension Agents indicated they use newsletters as a means of advertisement, where $118(96.8 \%)$ beef producers indicated they would prefer to receive newsletters as a form of advertisement (see Table 31). 
Sixty-eight (64.7\%) producers indicated they would prefer to learn of programs via radio, while 12 (36.4\%) of the agents indicated they use radio as a form of advertisement. One other source of advertisement was Internet. Seventy-one (68.9\%) of beef producers indicated they would prefer to receive advertisements about upcoming programs via the Internet, while $15(54.5 \%)$ agents indicated that they use the Internet as a method of advertisement (see Table 31).

When ask about television as a method of advertisement, nine (27.3\%) agents agreed to using television, while $71(68.9 \%)$ producers indicated they would prefer to receive information about upcoming programs via the television. Another method of advertisement was flyers. Ninety-six (87.3\%) producers indicated they prefer flyers as a form of advertisement, in agreement with the producers, 24 (72.7\%) agents indicated they used flyers as a form of advisement (see Table 31).

The final form of advertisement used for comparison was personal visits. Ninetysix $(86.4 \%)$ producers indicated they prefer personal visits as a source of advertisement, while, $27(81.8 \%)$ agents agreed in using personal visits as a method of advertisement (see Table 31). 
Table 31

Comparison of Advertisement Methods

\begin{tabular}{|c|c|c|c|c|c|c|c|c|}
\hline & \multicolumn{4}{|c|}{ Agents } & \multicolumn{4}{|c|}{ Producers } \\
\hline & \multicolumn{2}{|c|}{ Disagree } & \multicolumn{2}{|c|}{ Agree } & \multicolumn{2}{|c|}{ Disagree } & \multicolumn{2}{|l|}{ Agree } \\
\hline & $\mathrm{N}$ & $\%$ & $\mathrm{~N}$ & $\%$ & $\mathrm{~N}$ & $\%$ & $\mathrm{~N}$ & $\%$ \\
\hline Email & 17 & 50.0 & 17 & 50.0 & 30 & 33.3 & 60 & 66.7 \\
\hline Mail & 2 & 5.7 & 33 & 94.3 & 2 & 1.6 & 124 & 98.4 \\
\hline Phone & 7 & 20.0 & 28 & 80.0 & 32 & 29.3 & 77 & 70.7 \\
\hline $\begin{array}{l}\text { Word of } \\
\text { mouth }\end{array}$ & 1 & 2.9 & 34 & 97.1 & 27 & 24.1 & 85 & 75.9 \\
\hline Newspapers & 1 & 2.9 & 33 & 97.1 & 18 & 15.8 & 96 & 84.2 \\
\hline Newsletters & 6 & 18.2 & 27 & 81.8 & 4 & 3.3 & 118 & 96.8 \\
\hline Radio & 21 & 63.6 & 12 & 36.4 & 37 & 35.2 & 68 & 64.7 \\
\hline Internet & 18 & 54.5 & 15 & 45.5 & 31 & 33.4 & 62 & 66.7 \\
\hline Television & 24 & 72.7 & 9 & 27.3 & 32 & 31.0 & 71 & 68.9 \\
\hline Flyers & 9 & 27.3 & 24 & 72.7 & 14 & 12.7 & 96 & 87.3 \\
\hline $\begin{array}{l}\text { Personal } \\
\text { visits }\end{array}$ & 6 & 18.2 & 27 & 81.8 & 15 & 13.5 & 96 & 86.4 \\
\hline
\end{tabular}

Of the $128(100 \%)$ beef producers that responded to the study all of them agreed that they had a desire for the demonstration method, where as only 30 (88.2\%) Extension Agents agreed they used the demonstration method. One Hundred seventeen $(93.6 \%)$ beef producers had a desire to learn by the lecture method, where as only $30(88.2 \%)$ of Extension Agents indicated they used this method of teaching. The discussion method 
was by $123(97.6 \%)$ beef producers while 32 (94.1\%) Extension Agents agreed to using this method of teaching with their producers (see Table 32).

Table 32

Comparison of Teaching Methods

\begin{tabular}{|c|c|c|c|c|c|c|c|c|}
\hline & \multicolumn{4}{|c|}{ Producers } & \multicolumn{4}{|c|}{ Agents } \\
\hline & \multicolumn{2}{|c|}{ Disagree } & \multicolumn{2}{|c|}{ Agree } & \multicolumn{2}{|c|}{ Disagree } & \multicolumn{2}{|c|}{ Agree } \\
\hline & $\mathrm{N}$ & $\%$ & $\mathrm{~N}$ & $\%$ & $\mathrm{~N}$ & $\%$ & $\mathrm{~N}$ & $\%$ \\
\hline Demonstration & 0 & 0.0 & 128 & 100.0 & 4 & 11.8 & 30 & 88.2 \\
\hline Lecture & 8 & 6.4 & 117 & 93.6 & 4 & 11.8 & 30 & 88.2 \\
\hline Discussion & 3 & 2.4 & 123 & 97.6 & 2 & 5.9 & 32 & 94.1 \\
\hline Internet & 40 & 40.4 & 59 & 59.6 & 21 & 61.8 & 13 & 38.2 \\
\hline Fact Sheets & 8 & 7.1 & 105 & 92.9 & 3 & 8.8 & 31 & 91.2 \\
\hline $\begin{array}{l}\text { Showing video- } \\
\text { DVD }\end{array}$ & 10 & 8.4 & 108 & 91.6 & 5 & 14.7 & 29 & 85.3 \\
\hline $\begin{array}{l}\text { Teaching \&/or } \\
\text { demonstrating } \\
\text { computer } \\
\text { software }\end{array}$ & 30 & 29.7 & 71 & 70.3 & 22 & 66.7 & 11 & 33.3 \\
\hline Books & 20 & 17.9 & 92 & 82.1 & 18 & 54.5 & 15 & 45.5 \\
\hline $\begin{array}{l}\text { Individual } \\
\text { consultation }\end{array}$ & 7 & 5.8 & 114 & 94.2 & 3 & 9.1 & 30 & 90.9 \\
\hline
\end{tabular}

The Internet method was undesirable to twenty-one (61.8\%) agents, where as 59 $(59.6 \%)$ producers show a desire to learn by this method. Another teaching method was fact sheets. One hundred five $(91.6 \%)$ producers had a desire to learn by fact sheets and $31(91.2 \%)$ agents agreed to use this method of teaching. When beef producers were asked if showing a video or DVD was a desirable method of teaching, 108 (91.6\%) 
agreed, where $29(95.3 \%)$ agents agreed to using this method of teaching. West Virginia beef producers also had a desire to use books. Ninety-two $(82.1 \%)$ producers indicated a desire to use this method, while $15(45.5 \%)$ agents indicated they use this method of teaching. One other method of teaching is individual consultation. Thirty (90.9\%) agents agreed to using this method for teaching, and 114, (94.2\%) producers indicated they had a desire to learn by this method of teaching (see Table 32).

\section{Comparison of Extension Programming}

One hundred fifteen $(96.6 \%)$ producers indicated they would like to know more about the BQA program while $28(87.5 \%)$ agents indicated they offered this program. One hundred eight $(91.5 \%)$ producers indicated they were interested in the LRP program, where as $22(68.8 \%)$ agents indicated they offered the program (see Table 33$)$.

When asked about the replacement heifer program, $121(97.6 \%)$ producers indicated there was an interest; whereas 23(71.9\%) agents indicated they offered the program. In herd health management, $121(97.6 \%)$ producers indicated an interest, along with 29 (90.6\%) agents offering this program in their counties (see Table 33).

When the respondents came to reproduction fertility management, 117 (95.1\%) producers indicated an interest; twenty-six $(81.3 \%)$ agents offered this program. Another program was facility design. One hundred-fourteen $(95.8 \%)$ producers indicated an interest in this program, where as only 24 (75.0\%) agents offer this program (see Table 33). 
Table 33

Comparison of Extension Programming

\begin{tabular}{|c|c|c|c|c|c|c|c|c|}
\hline & \multicolumn{4}{|c|}{ Producers } & \multicolumn{4}{|c|}{ Agents } \\
\hline & \multicolumn{2}{|c|}{ Disagree } & \multicolumn{2}{|c|}{ Agree } & \multicolumn{2}{|c|}{ Disagree } & \multicolumn{2}{|c|}{ Agree } \\
\hline & $\mathrm{N}$ & $\%$ & $\mathrm{~N}$ & $\%$ & $\mathrm{~N}$ & $\%$ & $\mathrm{~N}$ & $\%$ \\
\hline $\begin{array}{l}\text { Beef Quality } \\
\text { Assurance } \\
\text { (BQA) }\end{array}$ & 4 & 3.3 & 115 & 96.6 & 4 & 12.5 & 28 & 87.5 \\
\hline $\begin{array}{l}\text { Livestock Risk } \\
\text { Protection } \\
\text { (LRP) }\end{array}$ & 10 & 8.4 & 108 & 91.5 & 10 & 31.3 & 22 & 68.8 \\
\hline $\begin{array}{l}\text { Replacement } \\
\text { heifer } \\
\text { management }\end{array}$ & 3 & 2.4 & 121 & 97.6 & 9 & 28.1 & 23 & 71.9 \\
\hline $\begin{array}{l}\text { Herd health } \\
\text { management }\end{array}$ & 1 & 0.8 & 128 & 99.2 & 3 & 9.4 & 29 & 90.6 \\
\hline $\begin{array}{l}\text { Reproduction- } \\
\text { fertility } \\
\text { management }\end{array}$ & 6 & 4.9 & 117 & 95.1 & 6 & 18.8 & 26 & 81.3 \\
\hline $\begin{array}{l}\text { Facilities } \\
\text { design }\end{array}$ & 5 & 4.2 & 114 & 95.8 & 8 & 25.0 & 24 & 75.0 \\
\hline Marketing & 5 & 4.0 & 121 & 96.1 & 4 & 12.1 & 29 & 87.9 \\
\hline $\begin{array}{l}\text { Record } \\
\text { keeping }\end{array}$ & 5 & 4.2 & 114 & 95.9 & 5 & 15.6 & 27 & 84.4 \\
\hline Nutrition & 2 & 1.6 & 122 & 98.4 & 3 & 9.1 & 30 & 90.9 \\
\hline $\begin{array}{l}\text { Forage } \\
\text { production \& } \\
\text { management }\end{array}$ & 3 & 2.5 & 119 & 97.6 & 3 & 9.1 & 30 & 90.9 \\
\hline $\begin{array}{l}\text { Genetic } \\
\text { evaluation }\end{array}$ & 6 & 5.1 & 111 & 94.9 & 7 & 21.9 & 25 & 78.1 \\
\hline Bull Test & 14 & 11.6 & 107 & 88.5 & 6 & 18.8 & 26 & 81.3 \\
\hline
\end{tabular}


When asked about a marketing program, $121(96.1 \%)$ producers indicated they were interested, where $29(84.4 \%)$ agents offered the program. Another program 119 (97.6\%) beef producers showed interest in was forage production \& management, 30 $(90.9 \%)$ agents agreed to they offer this program. When asked about genetic evaluation, $111(94.9 \%)$ producers indicated interest, where as $25(78.1 \%)$ agents indicated they offered the program in their counties. The final program used in the study was the bull tests. One hundred-seven $(88.5 \%)$ producers indicated they were interested in the program, while $26(81.3 \%)$ agents indicated they offered the program (see Table 33$)$. 


\section{CHAPTER V}

\section{Summary, Conclusions, Recommendations}

\section{Purpose and Objectives}

The purpose of this study was to determine whether information from the West Virginia University Extension Service being disseminated to the West Virginia beef producers through appropriate programming and advertising. The primary objective of this study was to determine whether the Extension Agents are offering the programs beef producers want in their counties. The research focused on program advertising, teaching methods, and program offerings.

\section{Research Questions}

The objectives of study were reflected in the following research questions:

1. What methods are West Virginia University Extension Agents using to inform beef producers about upcoming programs and new technologies relating to the beef industry?

2. How do beef producers prefer to hear about Extension programs?

3. What methods do West Virginia University Extension Agents use to teach their programs?

4. What are the methods by which West Virginia beef producers prefer to learn?

5. What beef production programs are West Virginia University Extension Agents offering in the state?

6. What programs would the beef producers like to see West Virginia University Extension Service offer in their counties?

7. Are West Virginia University Extension Agents effectively communicating with their county beef producers? 


\section{Summary}

The study consisted of 342 randomly selected West Virginia beef producers and a census of 46 agents responsible for Agriculture and Natural Resources in West Virginia, or agents in single agent counties. The response rates were $42 \%$ for beef producers and 87\% for Extension Agents.

Research Question 1. "What methods are West Virginia University Extension Agents using to inform beef producers about upcoming programs and new technologies relating to the beef industry?"

West Virginia University Extension Agents use a variety of methods to advertise for their programs. The top four methods of advertisement were word of mouth, newspapers, mail, and newsletters. Extension Agents least preferred method of advertising was television.

Research Question 2. "How do beef producers prefer to hear about Extension programs?"

West Virginia beef producers most preferred to receive information about upcoming Extension programs, by mail, newsletters, and flyers. The least preferred method for beef producers preferred was radio.

Research Question 3. "What methods do West Virginia University Extension Agents use to teach their programs?

When delivering beef programs to producers, Extension Agents' preferred methods were: discussion, fact sheets, and individual consultation. The least used teaching methods were books, Internet, and teaching or demonstrating computer software. 
Research Question 4. "What are the methods by which the West Virginia beef producers prefer to learn?"

The top teaching method most preferred by West Virginia beef producers was the demonstration method followed by lecture. The least preferred teaching method of West Virginia beef producers was teach or demonstrating computer software.

Research Question 5. "What beef production programs are the West Virginia Extension Agents offering in the state?"

The most frequently offered beef programs across the state by Extension Agents include: nutrition, forage production \& management, and reproduction-fertility management. The program least likely to be offered by Extension Agents across the state was Livestock Risk Protection.

Research Question 6. "What programs would the beef producers like to see the West Virginia University Extension Service offer in their counties?"

The top programs in which West Virginia beef producers were most interested in were: herd health, nutrition, and replacement heifer management. The program West Virginia beef producers were least interested in was the Livestock Risk Protection program.

Research Question 7. “Are West Virginia University Extension Agents connected with their beef producers in their counties?"

Although Extension Agents and beef producers top advertising methods were different, both groups included mail and newspapers in their top methods for advertisement. Radio and television were low on the preferred lists for both groups. 
Though there are differences between Extension Agents and beef producers, overall the groups appear to be unified with advertising methods.

Extension Agents and beef producers both selected the demonstration as their preferred method of teaching. Both groups also ranked teaching or demonstrating software low on the preferred list of teaching methods.

When it comes to programs the two groups were in disagreement. Extension Agents preferred to offer nutrition, forage, and reproduction management programs, while beef producers were more interested in herd health, nutrition, and replacement heifer management.

\section{Conclusion}

Based on the results of this study, the following conclusions were made

1. Extension Agents and beef producers both rate demonstration as their preferred teaching method.

2. A majority of the beef producers know if their Extension agent is responsible for one or more counties.

3. Extension Agents and West Virginia beef producers agree on preferred methods of advertisement for programs.

4. West Virginia beef producers agree that their WVU Extension Agents were able to answer their beef related questions.

5. West Virginia University Extension Agents offer all programs that beef producers indicate they have an interest in; however, the preferred programs differ between the two groups.

6. Beef producers do attend Extension beef related programs. 
7. The findings of this study were similar to that of Nelson (2008) with Extension Agents and dairy producers in Pennsylvania.

\section{Recommendations}

The researcher makes the following recommendations based on the results of this study:

1. West Virginia University Extension Agents should use the demonstration method as the main teaching method when working with beef producers.

2. Extension Agents should advertise for beef programs using mail, newsletters, word of mouth, and newspapers.

3. West Virginia Extension Agents should conduct a needs assessment in their counties to determine what type of beef programs producers are most interested in attending.

4. Extension Agents should conduct a study in their county to determine how beef producers are getting answers to their beef production questions, preferred methods of advertisement, and methods of program delivery.

5. Extension Agents should consider using radio and television where available as a source of advertisement or program delivery.

6. This study should be conducted with Extension Agents and other producer groups. 


\section{REFERENCES}

2007 Census of Agriculture (2007). Retrieved September 25, 2009 from http://www.agcensus.usda.gov/Publications/2007/Online_Highlights/County_Prof iles/West_Virginia/cp99054.pdf

Ary, D., Jacobs, C., Razavieh, A., Sorenson. (2006). Introduction to research in education. ( $7^{\text {th }}$ ed.). Belmont, CA: Thomson Wadsworth.

Batte, M. (2004). Changing computer use in agriculture: evidence from Ohio. Computers and Electronics in Agriculture, 47(1), 1-13.

Burrows, M. (2008) Using local farmers' markets to promote Extension programs. Journal of Extension. 46(6). Retrieved February 21, 2010 from http://www.joe.org/joe/2008december/iw1.php

Chizari, M., Karbasioun, M. \& Linder, J.R. (1998). Obstacles facing Extension Agents in the development and delivery of Extension educational programs for adult farmers in the province of Esfahan, Iran. Journal of Agricultural Education, 39(1), 48-54.

Cohen, J. (1988). Statistical power analysis for the behavioral sciences $\left(2^{\text {nd }}\right.$ ed.) Hillsdale, NJ: Lawrence Eribaum Associates.

Dillman, D.A. (2007). Mail and Internet surveys: The tailored design method ( $2^{\text {nd }}$ ed.). New Jersey: John Wiley.

Findlay, H., Zabawa, R., Morris, C., Oben, M. (1993). Computer awareness among Limited-Resource Farmers. Journal of Extension, 31(1). Retrieved September 16, 2009 from http://www.joe.org/joe/1993spring/a8.php

Gerber, J. (1992). Farmer participation in research: A model for adaptive research and education. American Journal of Alternative Agriculture, 7(3), 118-121.

Gamon, J., Harrold, N., \& Creswell, J. (1994). Educational delivery methods to encourage adoption of sustainable agricultural practices. Journal of Agricultural Education, 35(1), 38-42.

Habeeb, M., Birkenholz, R., \& Weston, C. (1987). Clientele group and Extension council officer perception of the Cooperative Agriculture Extension Service. Journal of American Association of Teacher Educators in Agriculture, 28(4). Retrieved December 7, 2009 from http://pubs.aged.tamu.edu/jae//pdf/vol28/28-04-15.pdf 
Houghton, H., Arrington, L., Bradshaw, J. (1994). Effectiveness of correspondence instruction in an Extension vegetable gardening program. Journal of Agriculture Education. 35(1). Retrieved September 15, 2009 from http://pubs.aged.tamu.edu/jae/pdf/Vol35/35-01-21.pdf

Iddings, R.K., Apps, J.W. (1990). What influences farmer's computer use? Journal of Extension, 28(1). Retrieved September 15, 2009 from http://www.joe.org/joe/1990spring/a4.php

Krejcie, R.V. \& Morgan, D.W. (1960). Determining sample size for research activities. Educational and Psychological Measurement, 30, 607-610.

National Agricultural Statistics Service (2007). Retrieved September 15, 2009 from http://www.agcensus.usda.gov/Publications/2007/Online_Highlights/County_Prof iles/West_Virginia/index.asp

National Institute of Food and Agriculture (2009). Retrieved November 22, 2009 from http://www.csrees.usda.gov/about/background.html

Neehouse, L. M. (2005). Mass communication delivery methods used and possessed by Extension Agents in West Virginia. Unpublished master's thesis, West Virginia University, Morgantown, West Virginia. Retrieved September 25, 2009 from http://wvuscholar.wvu.edu:8881//exlibris/dtl/d3_1/apache_media/7401.pdf

Nehiley, J.M. (2001). Developing a simple four-step marketing plan for Extension programs. Journal of Extension, 39(2). Retrieved September 25, 2009 from http://www.joe.org/joe/2001april/iw3.php

Nelson, C. (2008). Evaluation of information transfer between Extension Agents and dairy producers in Pennsylvania. Unpublished master's thesis, West Virginia University, Morgantown, West Virginia. Retrieved September 15, 2009 from http://wvuscholar.wvu.edu:8881//exlibris/dtl/d3_1/apache_media/13997.pdf

Peters, B.A. (1998). Measuring the quality of communication linkages between farmers and the agricultural agencies responsible for the diffusion of innovations in the south west province of Cameroon. Unpublished master's thesis, Texas A\&M University, College Station, TX. Retrieved November 22, 2009 from http://www.eric.ed.gov/

Rexroad, T. (2002). Evaluation of Marketing methods used to promote Extension programs as perceived by Master Gardeners in West Virginia. Unpublished master's thesis, West Virginia University, Morgantown, West Virginia . Retrieved September 15, 2009 from http://wvuscholar.wvu.edu:8881//exlibris/dtl/d3_1/apache_media/6167.pdf 
Richardson, J., \& Mustain, R. (1988). Preferred methods of delivery of technological information by the North Carolina Agricultural Extension Service: Opinions of agricultural procedures who uses Extension information. North Carolina: North Carolina State University. Retrieved September 26, 2008 from http://www.eric.ed.gov/ERICDocs/data/ericdocs2sq1/content_storage_01/0000019 b/80/1d/83/dc.pdf

Riesenberg, L. E., \& Gor, C. O. (1989). Farmers' preferences for methods of receiving information on new or innovative farming practices. Journal of Agricultural Education, 30(3), 7-13. Retrieved January 10, 2010 from http://pubs.aged.tamu.edu/jae//pdf/vol30/30-03-07.pdf

Robinson, J., Shaver P., Wrightsman, L. (1991). Measures of personality and social psychological attitudes. (pp 1-16) New York: Academic Press.

Seevers, B., Graham, D., \& Conklin, N. (2007). Education through cooperative Extension. ( ${ }^{\text {nd }}$ ed.). Columbus: Curriculum Materials Service. P 1-3.

Smith-Lever Act. Retrieved November 22, 2009 from http://www.highered.org/resources/smith.htm

Suvedi, M., Lapinski, M. K. \& Campo, S. (2000). Farmer's perspectives of Michigan State University Extension: trends and lessons from 1996 and 1999. Journal of Extension, 38(1). Retrieved January 15, 2010 from http://www.joe.org/joe/2000february/a4.html

Warner, P., Christenson, J., Dillman, D., \& Salant, P. (1996) Public perception of Extension. Journal of Extension, 34(4). Retrieved September 15, 2009 from http://joe.org/joe/1996august/a1.php 
APPENDICES 
APENDIX A

First Mailing Cover Letter

Extension Agent 
January 11,2010

\section{Dear West Virginia Extension Agent:}

As an Agent in West Virginia, I am sure you are aware that it takes many things to make a beef operation successful. At some point you have probably answered questions relating to beef production. The programs and advice you offer to beef producers in your county, make you a valuable resource.

I am Travis Cullen, a graduate student in Agricultural and Extension Education at West Virginia University. Under the direction of my advisor, Dr. Deborah Boone, I am conducting a research study to determine the amount and type of information transfer between county Extension Agents and beef producers in West Virginia. The result of this research study will be used to prepare a thesis to partially fulfill the requirements for a Master of Science degree in Agricultural and Extension Education. The result will be used to develop programs to better serve the beef producers of West Virginia. West Virginia University's IRB acknowledgement of this research is on file.

Your participation in this research study is solely voluntary. You may stop filling out this survey at any time or skip any question you do not wish to answer. However, your completion of this survey is crucial to the success of this study. The survey should only take about 15 minutes and your results will be held as confidential as possible. There is no penalty if you choose not to participate. You will notice a code number at the bottom left hand corner of your return envelope. This number is only used to keep track of non-respondents and will be separated from your survey and destroyed before the data is analyzed making it in no way possible to track your individual response.

Please place the completed questionnaire in the self addressed pre paid envelope and drop it into the mail box by January 27, 2010. Those responding by this date will receive an outdoor thermometer. For questions, you may contact Dr. Boone at 304-2935450 or Travis at 304-293-2743. Thank you, we sincerely appreciate your time and effort.

Sincerely,

Travis J. Cullen

Graduate Student
Deborah A. Boone, Ph.D.

Associate Professor 
APENDIX B

Second Mailing Cover Letter

Extension Agent 
January 29,2010

Dear West Virginia Extension Agent:

On January 11, we sent you a survey regarding your views on your beef programs. As of today we have not received your reply; we are sending a second copy of the survey and hope you will complete and return. If you have already returned the first survey there is no need to complete this one, we sincerely appreciate your participation.

As an Agent in West Virginia, I am sure you are aware that it takes many things to make a beef operation successful. At some point you have probably answered questions relating to beef production. The programs and advice you offer to beef producers in your county, make you a valuable resource.

I am Travis Cullen, a graduate student in Agricultural and Extension Education at West Virginia University. Under the direction of my advisor, Dr. Deborah Boone, I am conducting a research study to determine the amount and type of information transfer between county Extension Agents and beef producers in West Virginia. The result of this research study will be used to prepare a thesis to partially fulfill the requirements for a Master of Science degree in Agricultural and Extension Education. The result will be used to develop programs to better serve the beef producers of West Virginia. West Virginia University's IRB acknowledgement of this research is on file.

Your participation in this research study is solely voluntary. You may stop filling out this survey at any time or skip any question you do not wish to answer. However, your completion of this survey is crucial to the success of this study. The survey should only take about 15 minutes and your results will be held as confidential as possible. There is no penalty if you choose not to participate. You will notice a code number at the bottom left hand corner of your return envelope. This number is only used to keep track of non-respondents and will be separated from your survey and destroyed before the data is analyzed making it in no way possible to track your individual response.

Please place the completed questionnaire in the self addressed pre paid envelope and drop it into the mail box by February 12, 2010. For questions, you may contact Dr. Boone at 304-293-5450 or Travis at 304-293-2743. Thank you, we sincerely appreciate your time and effort.

Sincerely,

Travis J. Cullen

Graduate Student
Deborah A. Boone, Ph.D. Associate Professor 
APPENDIX C

First Mailing Cover letter

Beef Producer 
January 11,2010

Dear West Virginia Beef Producer:

As a producer in West Virginia, you are aware that it takes many things to make a beef operation successful. At some point in your career I am sure you have had questions about your farm. To answer these questions you may have turned to a fellow producer, a veterinarian, or county Extension Agent.

I am Travis Cullen, a graduate student in Agricultural and Extension Education at West Virginia University. Under the direction of my advisor, Dr. Deborah Boone, I am conducting a research study to determine the amount and type of information transfer between county Extension Agents and beef producers in West Virginia. The result of this research study will be used to prepare a thesis to partially fulfill the requirements for a Master of Science degree in Agricultural and Extension Education. The result will be used to develop programs to better serve the beef producers of West Virginia. West Virginia University's IRB acknowledgement of this research is on file.

Your participation in this research study is solely voluntary. You may stop filling out this survey at any time or skip any question you do not wish to answer. However, your completion of this survey is crucial to the success of this study. The survey should only take about 15 minutes and your results will be held as confidential as possible. There is no penalty or services withheld if you choose not to participate. You will notice a code number at the bottom left hand corner of your return envelope. This number is only used to keep track of non-respondents and will be destroyed before that data is analyzed making it in no way possible to track your individual response.

Please place the completed questionnaire in the self addressed pre paid envelope and drop it into the mail box by January 27, 2010. Those responding by this date will receive an outdoor thermometer. For questions, you may contact Dr. Boone at 304-2935450 or Travis at 304-293-2743. Thank you, we sincerely appreciate your time and effort.

Sincerely,

Travis J. Cullen

Graduate Student
Deborah A. Boone, Ph.D.

Associate Professor 
APPENDIX D

Second Mailing Cover letter

Beef Producer 
January 29,2010

Dear West Virginia Beef Producer:

On January 11 we sent you a survey regarding your views on your county's Extension agent and their beef programs. As of today we have not received your reply; we are sending a second copy of the survey and hope you will complete and return. If you have already returned the first survey there is no need to complete this one, we sincerely appreciate your participation.

As a producer in West Virginia, you are aware that it takes many things to make a beef operation successful. At some point in your career I am sure you have had questions about your farm. To answer these questions you may have turned to a fellow producer, a veterinarian, or county Extension Agent.

I am Travis Cullen, a graduate student in Agricultural and Extension Education at West Virginia University. Under the direction of my advisor, Dr. Deborah Boone, I am conducting a research study to determine the amount and type of information transfer between county Extension Agents and beef producers in West Virginia. The result of this research study will be used to prepare a thesis to partially fulfill the requirements for a Master of Science degree in Agricultural and Extension Education. The result will be used to develop programs to better serve the beef producers of West Virginia. West Virginia University's IRB acknowledgement of this research is on file.

Your participation in this research study is solely voluntary. You may stop filling out this survey at any time or skip any question you do not wish to answer. However, your completion of this survey is crucial to the success of this study. The survey should only take about 15 minutes and your results will be held as confidential as possible. There is no penalty or services withheld if you choose not to participate. You will notice a code number at the bottom left hand corner of your return envelope. This number is only used to keep track of non-respondents and will be destroyed before that data is analyzed making it in no way possible to track your individual response.

Please place the completed questionnaire in the self addressed pre paid envelope and drop it into the mail box by February 12, 2010. For questions, you may contact Dr. Boone at 304-293-5450 or Travis at 304-293-2743. Thank you, we sincerely appreciate your time and effort.

Sincerely,

Travis J. Cullen

Graduate Student
Deborah A. Boone, Ph.D. Associate Professor 
APPENDIX E

Extension Instrument 


\section{Evaluation of Information Transfer Between}

\section{Beef Producers and Extension Agents}

in West Virginia

\section{Extension Agent Survey}

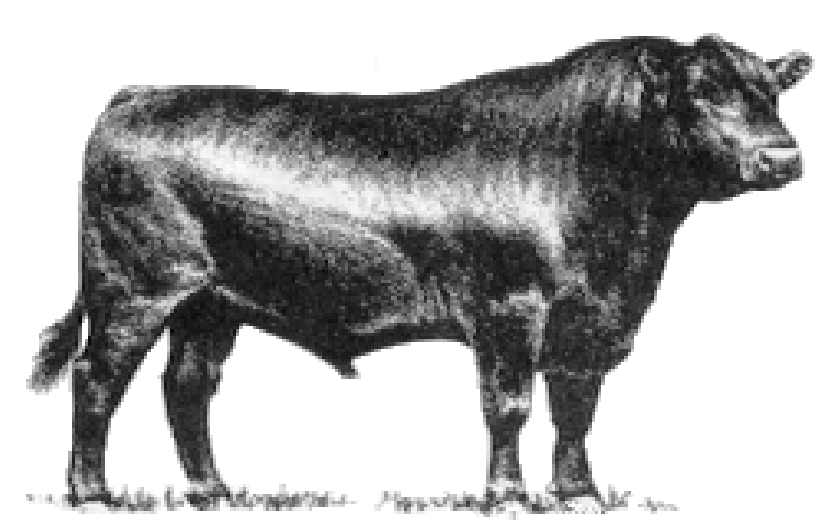

Travis Cullen

Agricultural and Extension Education

Davis College of Agriculture, Natural Resources, and Design

West Virginia University

26506 


\section{Evaluation of Information Transfer Between \\ Beef Producers and Extension Agents \\ in West Virginia}

Instructions: Using the following Likert scale, rate the following statements

about your county Extension office's agents and programs. Indicate your opinion by circling the letters that best corresponds to your response. SD Strongly Disagree, MDModerately Disagree, D- Disagree, A- Agree, MA Moderately Agree, SA- Strongly Agree.

\begin{tabular}{|c|c|c|c|c|c|c|}
\hline & 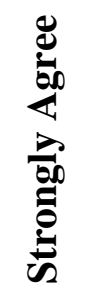 & 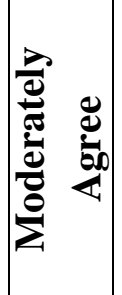 & ס્ّ & 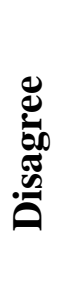 & 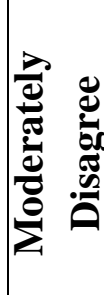 & 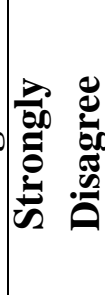 \\
\hline 1. I offer programs in beef production. & SA & MA & A & D & MD & SD \\
\hline $\begin{array}{l}\text { 2. I am able to answer most questions about beef } \\
\text { production. }\end{array}$ & SA & MA & A & D & MD & SD \\
\hline $\begin{array}{l}\text { 3. I return beef producers' telephone calls in a } \\
\text { timely manner. }\end{array}$ & SA & MA & A & D & MD & SD \\
\hline $\begin{array}{l}\text { 4. I return beef producers' emails in a timely } \\
\text { manner. }\end{array}$ & SA & MA & A & D & MD & SD \\
\hline $\begin{array}{l}\text { 5. Beef production program offered in my } \\
\text { county are well attended. }\end{array}$ & SA & MA & A & D & MD & SD \\
\hline $\begin{array}{l}\text { 6. The beef production practices taught are } \\
\text { readily accepted. }\end{array}$ & SA & MA & A & D & MD & SD \\
\hline $\begin{array}{l}\text { 7. I consider beef production one of my } \\
\text { specialty areas. }\end{array}$ & SA & MA & A & D & MD & SD \\
\hline
\end{tabular}




\begin{tabular}{|c|c|c|c|c|c|c|}
\hline & 趦 & 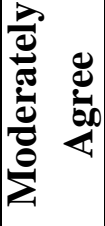 & 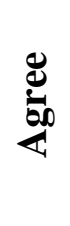 & 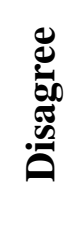 & 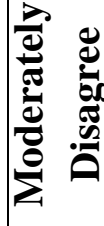 & 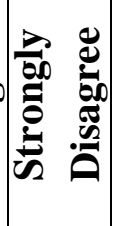 \\
\hline \multicolumn{7}{|c|}{ I use the following methods to advertise my beef programs. } \\
\hline 8. Email & SA & MA & A & $\mathrm{D}$ & MD & SD \\
\hline 9. Mail & SA & MA & A & $\mathrm{D}$ & MD & SD \\
\hline 10. Phone & SA & MA & A & $\mathrm{D}$ & MD & SD \\
\hline 11. Word of mouth & SA & MA & A & $\mathrm{D}$ & MD & SD \\
\hline 12. Newspapers & SA & MA & A & $\mathrm{D}$ & MD & SD \\
\hline 13. Newsletters & SA & MA & A & $\mathrm{D}$ & MD & SD \\
\hline 14. Radio & SA & MA & A & $\mathrm{D}$ & MD & SD \\
\hline 15. Internet & SA & MA & A & $\mathrm{D}$ & MD & SD \\
\hline 16. Television & SA & MA & A & $\mathrm{D}$ & MD & SD \\
\hline 17. Flyers & SA & MA & A & $\mathrm{D}$ & MD & SD \\
\hline 18. Personal visits & SA & MA & A & $\mathrm{D}$ & MD & SD \\
\hline $\begin{array}{l}\text { 19. Other (Please } \\
\text { specify }\end{array}$ & SA & MA & A & $\mathrm{D}$ & MD & SD \\
\hline $\begin{array}{l}\text { 20. Other (Please } \\
\text { specify }\end{array}$ & SA & MA & A & $\mathrm{D}$ & MD & SD \\
\hline
\end{tabular}




\begin{tabular}{|c|c|c|c|c|c|c|}
\hline & 党 & 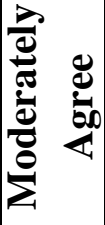 & & 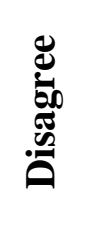 & 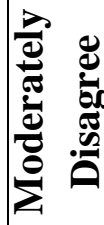 & 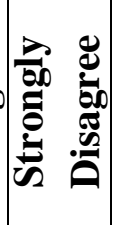 \\
\hline \multicolumn{7}{|c|}{ I use the following teaching methods to deliver beef programs. } \\
\hline 21. Demonstrations & SA & MA & A & $\mathrm{D}$ & MD & SD \\
\hline 22. Lectures & SA & MA & A & $\mathrm{D}$ & MD & SD \\
\hline 23. Discussion & SA & MA & A & $\mathrm{D}$ & MD & SD \\
\hline 24. Internet & SA & MA & A & $\mathrm{D}$ & MD & SD \\
\hline 25. Fact sheets & SA & MA & A & $\mathrm{D}$ & MD & SD \\
\hline 26. Showing video/DVD & SA & MA & A & $\mathrm{D}$ & MD & SD \\
\hline $\begin{array}{l}\text { 27. Teaching and/or demonstrating computer } \\
\text { software }\end{array}$ & SA & MA & A & $\mathrm{D}$ & MD & SD \\
\hline 28. Books & SA & MA & A & $\mathrm{D}$ & MD & SD \\
\hline 29. Individual Consultation & SA & MA & A & $\mathrm{D}$ & MD & SD \\
\hline $\begin{array}{l}\text { 30. Other (Please } \\
\text { specify }\end{array}$ & SA & MA & A & $\mathrm{D}$ & MD & SD \\
\hline $\begin{array}{l}\text { 31. Other (Please } \\
\text { specify }\end{array}$ & SA & MA & A & $\mathrm{D}$ & MD & SD \\
\hline
\end{tabular}




\begin{tabular}{|c|c|c|c|c|c|c|}
\hline & 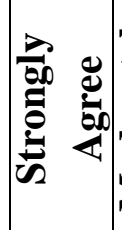 & 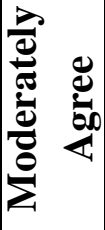 & 离 & 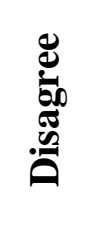 & 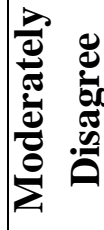 & 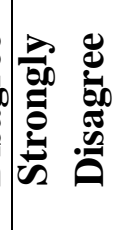 \\
\hline \multicolumn{7}{|l|}{ I offer the following beef programs } \\
\hline 32. Beef Quality Assurance [BQA] & SA & MA & A & $\mathrm{D}$ & MD & SD \\
\hline 33. Livestock Risk Protection [LRP] & SA & MA & A & $\mathrm{D}$ & MD & SD \\
\hline 34. Replacement heifer management & SA & MA & A & $\mathrm{D}$ & MD & SD \\
\hline 35. Herd health management & SA & MA & A & $\mathrm{D}$ & MD & SD \\
\hline 36. Reproduction/fertility management & SA & MA & A & $\mathrm{D}$ & MD & SD \\
\hline 37. Facilities design & SA & MA & A & $\mathrm{D}$ & MD & SD \\
\hline 38. Marketing & SA & MA & A & $\mathrm{D}$ & MD & SD \\
\hline 39. Record keeping & SA & MA & A & $\mathrm{D}$ & MD & SD \\
\hline 40. Nutrition & SA & MA & A & $\mathrm{D}$ & MD & SD \\
\hline 41. Forage production \& management & SA & MA & A & $\mathrm{D}$ & MD & SD \\
\hline 42. Genetic evaluation & SA & MA & A & $\mathrm{D}$ & MD & SD \\
\hline 43. Bull Test & SA & MA & A & $\mathrm{D}$ & MD & SD \\
\hline $\begin{array}{l}\text { 44. Other (Please } \\
\text { specify }\end{array}$ & SA & MA & A & $\mathrm{D}$ & MD & SD \\
\hline $\begin{array}{l}\text { 45. Other (Please } \\
\text { specify }\end{array}$ & SA & MA & A & $\mathrm{D}$ & MD & SD \\
\hline
\end{tabular}


Instructions: Answer the following questions to the best of your ability.

46. How many beef programs do you offer per year? (Check one)

a. 0

b. $1-2$

c. $3-4$

d. 5 or more

47. Beef programs account for $\%$ of my yearly programming. (Check one)

a. $0-25$

b. $26-50$

c. $51-75$

d. 76-more

48. On average how many beef producers do you work within a year?

a. $0-10$

b. $11-20$

c. $21-30$

d. $31-40$

e. $41-50$

f. $51-60$

g. $61-70$

h. $71-80$

i. $81-90$

j. $91-100$

j. 101 or more 
49. What is the average beef herd size per farm in your county? (Check one)

a. $1-20$

b. $21-40$

c. $41-60$

d. $61-80$

e. $81-100$

f. 101 or more

50. Which of the following best describes your county's beef cattle operations? (Check all that apply)

a. Commercial cow/calf selling feeder cattle

b. Commercial cow/calf retaining ownership through slaughter

c. Seedstock

d. Stocker operations sell as yearlings

e. Feedlot

f. Other:

51. What is the average age of beef producers within your county? (Check one)

a. Younger than 20 years

b. 20-29 years

c. 30-39 years

d. $40-49$ years

e. $50-59$ years

f. 60 or older 
52. What are the average years of operation for your beef cattle producers?
a. Less than 1 year
b. $1-5$ years
c. 6-10 years
d. $11-15$ years
e. $16-20$ years
f. 21-25 years
g. 26 or more

53. Where do you feel that beef producers in your county get answers to their beef production questions? (Rank all with 1 being the most used, 2 being the second most used and 3 being the third most used, etc.)
a. WVU Extension Agent
b. Extension Specialist
c. Veterinarian
d. Neighbor
e. Farm Store Employee
f. Other: 


\section{Comments:}

If you have any questions regarding this survey feel free to contact: Travis by phone at 304-293-6131 ext. 4234 or email: tcullen1@mix.wvu.edu or Dr. Debby Boone at 304-293-5450 or email at debby.boone@mail.wvu.edu Thank you for taking the time and effort to complete this survey. 
APPENDIX F

Beef Producer Instrument 


\section{Evaluation of Information Transfer Between}

\section{Beef Producers and Extension Agents}

in West Virginia

\section{Beef Producer Survey}

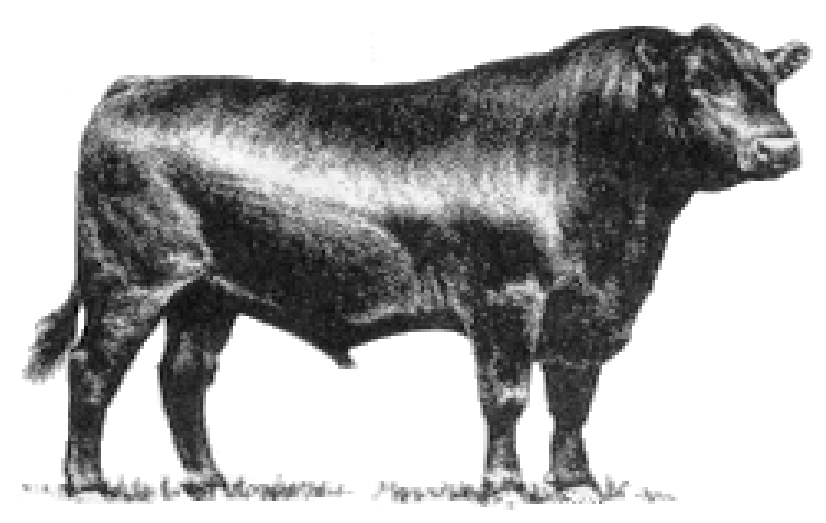

Travis Cullen

Graduate Student

Agricultural and Extension Education

Davis College of Agriculture, Natural Resources, and Design

West Virginia University

Morgantown, West Virginia, 26506 


\section{Evaluation of Information Transfer Between \\ Beef Producers and Extension Agents \\ in West Virginia}

Instructions: Using the following Likert scale, rate the following statements about local Extension Agents and programs. Indicate your opinion by circling the letters that best corresponds to your response. SA- Strongly Agree, MA- Moderately Agree, A - Agree, D - Disagree, MD - Moderately Agree, SD Strongly Disagree, or NA - Not Applicable.

\begin{tabular}{|l|l|l|l|l|l|l|l|}
\hline & & & & & & & \\
\hline $\begin{array}{l}\text { 46. The local West Virginia U Extension } \\
\text { Office offers programs in beef production. }\end{array}$ & $\mathrm{SA}$ & $\mathrm{MA}$ & $\mathrm{A}$ & $\mathrm{D}$ & $\mathrm{MD}$ & $\mathrm{SD}$ & $\mathrm{NA}$ \\
\hline $\begin{array}{l}\text { 47. The local West Virginia U Extension Agent } \\
\text { is able to answer most of my questions } \\
\text { about beef production. }\end{array}$ & $\mathrm{SA}$ & $\mathrm{MA}$ & $\mathrm{A}$ & $\mathrm{D}$ & $\mathrm{MD}$ & $\mathrm{SD}$ & $\mathrm{NA}$ \\
\hline $\begin{array}{l}\text { 48. The local West Virginia U Extension Agent } \\
\text { returns my telephone calls in a timely } \\
\text { manner. }\end{array}$ & $\mathrm{SA}$ & $\mathrm{MA}$ & $\mathrm{A}$ & $\mathrm{D}$ & $\mathrm{MD}$ & $\mathrm{SD}$ & $\mathrm{NA}$ \\
\hline $\begin{array}{l}\text { 49. The local West Virginia U Extension Agent } \\
\text { returns my emails in a timely manner. }\end{array}$ & $\mathrm{SA}$ & $\mathrm{MA}$ & $\mathrm{A}$ & $\mathrm{D}$ & $\mathrm{MD}$ & $\mathrm{SD}$ & $\mathrm{NA}$ \\
\hline $\begin{array}{l}\text { 50. I attend Extension beef programs offered in } \\
\text { my county. }\end{array}$ & $\mathrm{SA}$ & $\mathrm{MA}$ & $\mathrm{A}$ & $\mathrm{D}$ & $\mathrm{MD}$ & $\mathrm{SD}$ & $\mathrm{NA}$ \\
\hline $\begin{array}{c}\text { 51. Extension beef programs provide adequate } \\
\text { information to make an informed decision } \\
\text { on new practices. }\end{array}$ & $\mathrm{SA}$ & $\mathrm{MA}$ & $\mathrm{A}$ & $\mathrm{D}$ & $\mathrm{MD}$ & $\mathrm{SD}$ & $\mathrm{NA}$ \\
\hline $\begin{array}{c}\text { 52. I consider the local Extension agent to be } \\
\text { knowledgeable about beef production. }\end{array}$ & $\mathrm{SA}$ & $\mathrm{MA}$ & $\mathrm{A}$ & $\mathrm{D}$ & $\mathrm{MD}$ & $\mathrm{SD}$ & $\mathrm{NA}$ \\
\hline
\end{tabular}


Instructions: Using the following Likert scale, rate the following methods of advertisement. The first set is what you prefer, and the last set is what you receive from your local Extension. Indicate your opinion by circling the letters that best corresponds to your response. SA- Strongly Agree, MA- Moderately Agree, A - Agree, D - Disagree, MD - Moderately Agree, or SD Strongly Disagree.

\begin{tabular}{|c|c|c|c|c|c|c|c|c|c|c|c|c|}
\hline & 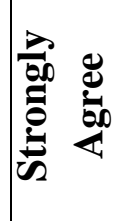 & 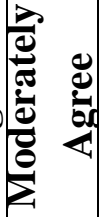 & 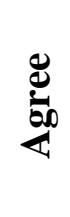 & 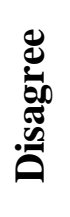 & 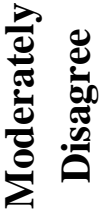 & 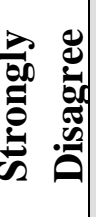 & 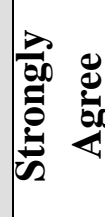 & لّيّ & 离 & 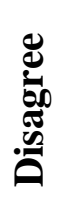 & 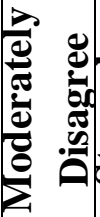 & 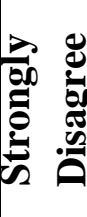 \\
\hline & \multicolumn{6}{|c|}{$\begin{array}{l}\text { I prefer the following methods } \\
\text { of advertisement for beef } \\
\text { programs: }\end{array}$} & \multicolumn{6}{|c|}{$\begin{array}{l}\text { I receive the following } \\
\text { methods of advertisement for } \\
\text { beef programs: }\end{array}$} \\
\hline 53.Email & SA & MA & A & $\mathrm{D}$ & MD & SD & SA & MA & A & D & MD & SD \\
\hline 54. Mail & SA & MA & A & $\mathrm{D}$ & MD & SD & SA & MA & A & $\mathrm{D}$ & MD & SD \\
\hline 55.Phone & SA & MA & A & $\mathrm{D}$ & MD & SD & SA & MA & A & $\mathrm{D}$ & MD & SD \\
\hline 56. Word of mouth & SA & MA & A & D & MD & SD & SA & MA & A & $\mathrm{D}$ & MD & SD \\
\hline 57. Newspapers & SA & MA & A & D & MD & SD & SA & MA & A & D & MD & SD \\
\hline 58. Newsletters & SA & MA & A & $\mathrm{D}$ & MD & SD & SA & MA & A & $\mathrm{D}$ & MD & SD \\
\hline 59. Radio & SA & MA & A & D & MD & SD & SA & MA & A & $\mathrm{D}$ & MD & SD \\
\hline 60. Internet & SA & MA & A & D & MD & SD & SA & MA & A & $\mathrm{D}$ & MD & SD \\
\hline 61. Television & SA & MA & A & $\mathrm{D}$ & MD & SD & SA & MA & A & $\mathrm{D}$ & MD & SD \\
\hline 62. Flyers & SA & MA & A & D & MD & SD & SA & MA & A & $\mathrm{D}$ & MD & SD \\
\hline 63. Personal visits & SA & MA & A & $\mathrm{D}$ & MD & SD & SA & MA & A & $\mathrm{D}$ & MD & SD \\
\hline \multicolumn{13}{|l|}{$\begin{array}{l}\text { 64. Other (Please } \\
\text { specify }\end{array}$} \\
\hline ) & SA & MA & A & D & MD & SD & SA & MA & A & $\mathrm{D}$ & MD & SD \\
\hline \multicolumn{13}{|l|}{$\begin{array}{l}\text { 65. Other (Please } \\
\text { specify }\end{array}$} \\
\hline & SA & MA & A & D & MD & SD & SA & MA & A & D & MD & SD \\
\hline
\end{tabular}


Instructions: Using the following Likert scale, rate the following teaching methods you would prefer your local Extension agent to use when teaching their programs. Indicate your opinion by circling the letters that best corresponds to your response. SA- Strongly Agree, MA- Moderately Agree, A - Agree, D - Disagree, MD - Moderately Agree, or SD Strongly Disagree.

\begin{tabular}{|c|c|c|c|c|c|c|}
\hline & 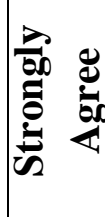 & 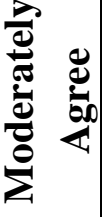 & 岁 & 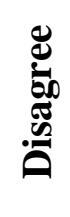 & 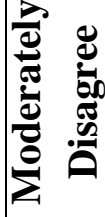 & 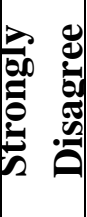 \\
\hline \multicolumn{7}{|c|}{ I prefer the following teaching methods at Extension programs: } \\
\hline 66. Demonstrations & SA & MA & A & $\mathrm{D}$ & MD & SD \\
\hline 67. Lectures & SA & MA & A & $\mathrm{D}$ & MD & SD \\
\hline 68. Discussion & SA & MA & A & $\mathrm{D}$ & MD & SD \\
\hline 69. Internet & SA & MA & A & $\mathrm{D}$ & MD & SD \\
\hline 70. Fact sheets & SA & MA & A & $\mathrm{D}$ & MD & SD \\
\hline 71. Showing video/DVD & SA & MA & A & $\mathrm{D}$ & MD & SD \\
\hline $\begin{array}{l}\text { 72. Teaching and/or demonstrating computer } \\
\text { software }\end{array}$ & SA & MA & A & $\mathrm{D}$ & MD & SD \\
\hline 73. Books & SA & MA & A & $\mathrm{D}$ & MD & SD \\
\hline 74. Individual consultation & SA & MA & A & $\mathrm{D}$ & MD & SD \\
\hline $\begin{array}{l}\text { 75. Other (Please } \\
\text { specify }\end{array}$ & SA & MA & A & $\mathrm{D}$ & MD & SD \\
\hline $\begin{array}{l}\text { 76. Other (Please } \\
\text { specify }\end{array}$ & SA & MA & A & D & MD & SD \\
\hline
\end{tabular}


Instructions: Using the following Likert scale, rate the following programs you would be interested in learning about. Indicate your opinion by circling the letters that best corresponds to your response. SA- Strongly Agree, MA- Moderately Agree, A - Agree, D - Disagree, MD - Moderately Agree, or SD Strongly Disagree .

\begin{tabular}{|c|c|c|c|c|c|c|}
\hline & 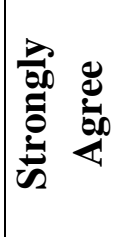 & 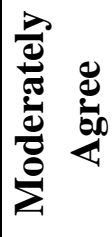 & 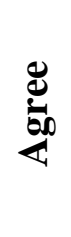 & 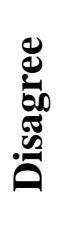 & 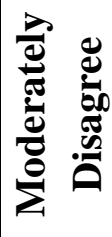 & 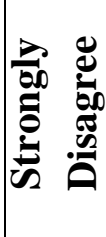 \\
\hline \multicolumn{7}{|l|}{ I am interested in beef programs related to: } \\
\hline 77. Beef Quality Assurance (BQA) & SA & MA & A & $\mathrm{D}$ & MD & SD \\
\hline 78. Livestock Risk Protection (LRP) & SA & MA & A & $\mathrm{D}$ & MD & SD \\
\hline 79. Replacement heifer management & SA & MA & A & $\mathrm{D}$ & MD & SD \\
\hline 80. Herd health management & SA & MA & A & $\mathrm{D}$ & MD & SD \\
\hline 81. Reproduction/fertility management & SA & MA & A & $\mathrm{D}$ & MD & $\mathrm{SD}$ \\
\hline 82. Facilities design & SA & MA & A & $\mathrm{D}$ & MD & SD \\
\hline 83. Marketing & SA & MA & A & $\mathrm{D}$ & MD & SD \\
\hline 84. Record keeping & SA & MA & A & $\mathrm{D}$ & MD & SD \\
\hline 85. Nutrition & SA & MA & A & $\mathrm{D}$ & MD & $\mathrm{SD}$ \\
\hline 86. Forage production $\&$ management & SA & MA & A & $\mathrm{D}$ & MD & SD \\
\hline 87. Genetic evaluation & SA & MA & A & $\mathrm{D}$ & MD & SD \\
\hline 88. Bull Test & SA & MA & A & $\mathrm{D}$ & MD & SD \\
\hline $\begin{array}{l}\text { 89. Other (Please } \\
\text { specify }\end{array}$ & SA & MA & A & $\mathrm{D}$ & $\mathrm{MD}$ & SD \\
\hline $\begin{array}{l}\text { 90. Other (Please } \\
\text { specify }\end{array}$ & SA & MA & A & $\mathrm{D}$ & $\mathrm{MD}$ & $\mathrm{SD}$ \\
\hline
\end{tabular}


Instructions: Answer the following questions to the best of your ability.

91. How many beef Extension programs do you attend per year? (Check one)

a. 0

b. $1-2$

c. $3-4$

d. 5 or more

92. Beef Extension programs account for year? (Check one)

a. $0-25$

b. $26-50$

c. $51-75$

d. 76 or more

93. How many head of beef cattle do you have on your farm? (Check one)

a. $1-20$

b. $21-40$

c. $41-60$

d. $61-80$

e. $81-100$

f. 101 or more 
49. Which of the following best describes your beef cattle operation? (Check all that apply)

a. Commercial cow/calf selling feeder cattle

b. Commercial cow/calf retaining ownership through slaughter

c. Seedstock

d. Stocker operations sell as yearlings

e. Feedlot

f. Other:

50. What is your age range? (Check one)

a. Younger than 20 years

b. 20-29 years

c. 30-39 years

d. $40-49$ years

e. 50-59 years

f. 60 or older

51. Where do you get answers to your beef production questions? (Rank all with 1 being the most used, 2 being the second most used and 3 being the third most used, etc.)

a. WVU Extension Agent

b. Extension Specialist

c. Veterinarian

c. Neighbor 


\section{d. Farm Store Employee}

e. Other:

52. How many years has this operation been in the cattle business?

a. Less than 1 year

b. 1-5 years

c. 6-10 years

d. 11-15 years

e. $16-20$ years

f. 21-25 years

g. 26 or more

53. The local Extension agent, who handles beef programming...

a. has responsibility for my county only.

b. shares responsibility for two or more counties.

c. I am not sure 


\section{Comments:}

If you have any questions regarding this survey feel free to contact: Travis by phone at 304-293-6131 ext. 4234 or email: tcullen1@mix.wvu.edu or Dr. Debby Boone at 304-293-5450 or email at debby.boone@mail.wvu.edu

Thank you for taking the time and effort to complete this survey. 
APPENDIX G

Extension Agent Open Ended Responses 
Question 19: "I use the following methods to advertise my beef programs.”

Responses:

Regional Beef Newsletter

FSA Newsletter

Question 30: "I use the following teaching methods to deliver beef programs."

Responses:

Field Days

Farm Visits

One-on-One

Newsletters

Question 50: “The following best describes your county's beef cattle operation.”

Responses:

Hobby Farms

Grass Fed

Question 53: "Where do you feel that beef producers in your county get answers to their beef related questions?”

USDA

Breed Associations

NRCS

FSA

Other Farmers

Magazines

RFDTV

Internet 
APPENDIX H

Beef Producer Open Ended Responses 
Question 19: “I prefer/receive the following methods of advertisement for beef programs.”

Responses:

FFA Alumni News

Farm Tours

Meetings w/me and others

Field Days

Question 30: “I prefer the following teaching methods at extension programs”

Responses:

Multi County Meetings

Field Trips

Question 49: “Which of the following best describes your beef cattle operation?" Responses:

back grounding

Breeding

clean land

club calf production

club calf production

Cow/Calf Operation

Purebred Angus

purebred

Sell Some Bulls and Heifer Yearlings 
Question 51: “Where do you get answer to your beef production questions?”

Research

Internet

Ag Teachers

Trial and Error

Buyers

Books

American Angus Association

Fellow Farmers

Other Farmers

Other State Universities

Family Members 
APPENDIX I

\section{Extension Agent Comments}




\section{Comments:}

[There] is no "Ag agent in this county anymore. Any agriculture questions are answered by the office. No additional programs are offered. Agriculture related articles are put in the county Extension newsletter when available from state office. County offers an agriculture farm show where producers can show their beef. We also support the Beef Expo and West Virginia Livestock Round Up.

I would like to see the Extension Summary when finished. Good Luck. [county agent]

Do not offer programs at this time.

We have a local Cattleman Association that promotes beef quality and marketing; however, most beef farmers choose not to participate. I believe they're well aware of it and well aware of educational opportunities. They just don't /wont' make the effort to adopt best practices on their farm or get involved.

Main Educational Teaching through articles in Bi-Monthly newsletters. Others include Phone call, Farm Visit, Internet Links, meetings in Neighboring States Dinner Educational Meeting.

We have no Ag Agent or Beef program.

They often get answers from neighbors or the farm store because of more regular contact. But not necessarily the best answer.

Keep up the good work young man!! Do not give up! Stay focus. I wish you my best. [County Agent]

Very little beef production most people do it more so as a hobby and will slaughter to provide their family with beef. They will also go to the auction from time to time but not for large scale or purchase.1-3 animals at a time at the most.

I just started late in the year, so take this information with that in mind.

Good Questions Makes me realize how many more programs I need to be offering!. Good luck with the thesis. 
APPENDIX J

Beef Producer Comments 


\section{Comments:}

Calf pools are talked up but over the years local markets are the best bet. Sell in farmers groups.

Extension Agents seem to be in meetings in Morgantown or other places than their county, $75 \%$ of the time you need him or call office he is gone.

Get a Toll Free Number

Good Luck Bleed Green

He is Not Very User Friendly

I cannot see how this is going to result in better programs for farmers. Another example of a poor Extension Program

I definitely think the agent is doing a great job - just not knowledgeable in farming

I don't have a computer

I don't have email.

I go to a different County

I would like to see more development of the beef marketing program for W.V. I am not referring to the role of feeder cattle to out of state markets.

If you call our Extension Agent they try to be very helpful

I'm going out of business you can't make it. There is no market

In [West Virginia] County there are no beef classes at ALL.

I've used the agent one time in 11 years

More on organic Animal and crop production low inputs how to obtain more info on Herford cattle

[West Virginia] County, has not had an "agricultural" agent for years now. The last few have been great with $4-\mathrm{H}$ programs but no help with farming.

Our county Agent is always available when called as need arises

Thanks For a chance for Farmers to be heard. The financial challenges that the small farmers have today are unbelievable. If I have to borrow Money to Buy Fertilizer this year, and right now it looks like this is going to happen, I'll have to go out of business. How many small farms go out each year? Something has to change! Thanks 
The County agent of [West Virginia] County does a very good job of working with the cattle owners.

There is a feeder market in the [West Virginia City] area. The state should assist this development and keep those jobs in West Virginia. Call me after 6 pm and I will explain what I think will help the West Virginia Beef Producers

We have 232 Dinner meetings per year. They are on the most part very full of info.

We would like to have an organized sale of cattle of all breeds in our county

[West Virginia] County does not have any beef programs. We have to go to [West Virginia] County.

[West Virginia] County has a new agent.

West Virginia Bull Sale is not pushed due to change in grading several years ago.

Genetic Alliance Bull Sale is highly recommended but only two farms are involved along with Extension agent. 
VITA

Travis Cullen

Education: May 2010

December 2008

Profession

Experience:

May 2008 - August 2008

February 2009 -August 2009

August 2009 -May 2010

June 2010
Masters of Science

Agricultural and

Extension Education

West Virginia University

Morgantown, West Virginia

Bachelors of Science

Agra-business Management and

Rural Development

West Virginia University

Morgantown, West Virginia
Intern

WVU Extension Service West Virginia University

Point Pleasant, West Virginia

Graduate Student

WVU Extension Service

West Virginia University

Morgantown, West Virginia

Graduate Research Assistant WV Small Farm Center WVU Extension Service West Virginia University Morgantown, West Virginia

Extension Agent in Training WVU Extension Service West Virginia University Morgantown, West Virginia 